\title{
Invariant Background Field Method for Chiral Lagrangians Including Wess-Zumino Terms
}

\author{
R. Akhoury and A. Alfakih \\ Randall Lab of Physics, University of Michigan, \\ Ann Arbor, Michigan 48109-1120
}

Received October 20, 1990

\begin{abstract}
A chiral invariant background field method is introduced in order to study the structure of higher order terms in chiral perturbation theory. Explicit one-loop calculations are presented together with the construction of the most general $\mathcal{O}\left(p^{6}\right)$ chiral lagrangian relevant for anomalous processes. The non-renormalization of the coefficient of the Wess-Zumino term in both two and four dimensions is discussed in general. 1991 Academic Press. Inc.
\end{abstract}

\section{INTRODUCTION}

In recent years, chiral perturbation theory has received much attention as a means of describing the interactions of the light hadrons at low energy. In a series of papers Gasser and Leutwyler [1] have discussed in a systematic manner the basic features of chiral perturbation theory that can be used to calculate the generating functional of Green's functions of quark currents in an expansion in powers of external momenta $\left(p^{2}\right)$ and quark masses $(m)$. For this purpose an effective Lagrangian involving the pseudoscalar meson degrees of freedom is introduced. This effective chiral lagrangian is constructed so as to incorporate the symmetries, the assumed symmetry breaking pattern, and the anomalies of QCD. At lowest order in $p^{2}$ and quark masses (quark masses are counted as two chiral powers, consistent with chiral symmetry) the effective lagrangian is given by a non-linear sigma model coupled to cxternal fields which may be flavor non-singlet. The form of the chiral Lagrangian at higher orders is determined by symmetry requirements and in general an infinite number of terms with associated coupling constants containing structures of increasingly higher order in derivatives and quark masses allowed. In practice, however, one truncates at a given order (in derivatives and quark masses) and the coupling constants can be extracted at that order from experiments. Such a procedure for chiral $S U(3)$ was discussed in detail in Ref. [1] for the $\mathcal{O}\left(p^{4}\right)$ terms with even number of meson fields where there are 10 coupling constants $L_{1}, \ldots, L_{10}$. That these higher order terms are needed becomes obvious from the fact that meson loops need to be considered in order for the low energy expansion to be consistent with unitarity and that the non-linear sigma model in four dimensions is non-renormalizable. At the $\mathscr{C}\left(p^{4}\right)$ level, one must also include in the chiral 
lagrangian the Wess-Zumino terms which are odd in meson fields and which correctly incorporate the anomalies of QCD in the presence of external vector and axial vector fields.

Since the original work of Wess and Zumino [2] in which the anomalous lagrangian was directly obtained by integrating the Bardeen expression for the anomaly, Witten [3] has obtained a compact form for this lagrangian. Witten has shown that the Wess-Zumino lagrangian can be written in terms of the chiral field $U$, in a compact manner as an action defined on a five-dimensional surface whose boundary is four-dimensional space-time. Witten also showed, using topological arguments that the coefficient of this term is quantized and phenomenologically, in order to obtain the correct $\pi^{0} \rightarrow \gamma \gamma$ decay rate, this coefficient must be the number of colors, $N_{c}$. The form of the Wess-Zumino lagrangian including external vector and axial vector fields has been discussed extensively in the literature [3-5].

The next terms in the chiral counting are of sixth order and are of two different kinds. Those which are even in meson fields (non-anomalous sector) and those that are odd (anomalous sector). The terms even in meson fields are the most general structures consistent with chiral symmetry, some of which are needed to cancel the two-loop infinities generated by the $\mathscr{O}\left(p^{2}\right)$ lagrangian or the one-loop infinities with one vertex $\mathcal{O}\left(p^{2}\right)$ and the other $\mathcal{O}\left(p^{4}\right)$. More interesting is the case of the sixth-order terms that are odd in meson fields. Typically, these would be relevant in determining the radiative corrections to anomalous processes like $\pi^{0} \rightarrow \gamma \gamma$, $\eta \rightarrow \pi^{+} \pi^{-} \gamma$, etc. Some of these will be needed to cancel the infinities arising from one-loop diagrams with one normal vertex of order $p^{2}$ and an anomalous vertex of order $p^{4}$. In this connection, it is very important to ensure that the coefficient of the $\mathscr{O}\left(p^{4}\right)$ Wess-Zumino term is not renormalized. This would be the counterpart of the Adler-Bardeen theorem in the infrared regime and it is important to verify the non-renormalization of this Wess-Zumino coefficient to all orders in chiral perturbation theory.

The above discussion makes clear the need to have a systematic procedure for studying the structure of the higher order terms in chiral perturbation theory, both from a phenomenological and from a formal viewpoint. In order to do this, chiral invariant procedures have to be introduced to study the loop effects at higher orders. In the past, background field methods with external fields have been considered $[1,6,7]$ and a straightforward extension of these to higher order is computationally forbidding. Other background field methods relevant to the nonlinear sigma model have also been previously discussed $[8,9]$. In this paper, in particular Section II, we introduce a new coordinate independent background field expansion which is particularly well suited for the study of chiral lagrangians. This expansion is based on using geodesic curves on the group manifold of the chiral fields $U(x)$, to connect the background field and the chiral field, including background and fluctuations. The $\mathcal{O}\left(p^{2}\right)$ chiral lagrangian is first studied using this and a simple compact expression for the fluctuations to all orders is obtained. Next the Wess-Zumino lagrangian in the Witten form is considered. Here it is shown that the action incorporating the fluctuations to all orders can always be written in 
terms of the chiral field in a four-dimensional form and simple closed form expressions are again obtained. The case of two dimensions is also discussed, in particular, the conformally invariant two-dimensional Wess-Zumino model. Here, the advantage of our methods over the previous ones is very transparent. Certain structures that naturally appear in this background field expansion and have welldefined transformation properties under chiral $S U(3) \times S U(3)$ and/or the vector subgroups $S U(3)_{V}$ are identified. The local higher order terms in chiral perturbation theory must be constructed out of these structures. Finally, we conclude Section II with a discussion of how perturbation theory may be developed for the background field expanded actions.

In Section III we present some one-loop calculations to elucidate our method and also to bring out the rather simple looking expressions for the one-loop infinilies when expressed in terms of the (natural) basic structures introduced by our background field expansion. First, the order $p^{4}$ infinities are reproduced in agreement with earlier results, $[1,6,7]$, then the $\mathcal{O}\left(p^{6}\right)$ infinities in the anomalous sector are obtained. Finally, using symmetry considerations and certain identities relevant to four dimensions we construct the most general chiral lagrangian which is sixth order in the chiral counting. This sixth-order lagrangian contains external vector and axial vector fields, in general. There are 30 independent structures to this order with an associated number of new coupling constants. The non-renormalization of the Wess-Zumino coefficient is explicitly verified by direct calculations at the one-loop level.

In Section IV, we present a general discussion of the structure of the higher order terms in chiral perturbation theory. First, the non-renormalization of the Wess-Zumino coefficient in the two-dimensional model is discussed. The proof here is rather more straightforward than the ones discussed in the literature [9]. Next, we present arguments for the four-dimensional case for the non-renormalization of the coefficient of the Wess-Zumino term. We then conclude with a discussion of the results obtained in this paper.

\section{A Background Field Expansion for the Chiral Lagrangian}

In this section we will formulate a coordinate independent background field expansion for the non-linear sigma model both with an without the Wess-Zumino terms. This method will be seen to be more convenient than the usual expansion $[8,9]$, in a coordinate basis, especially in regard to the higher loop effects. We will then discuss how perturbation theory is developed for the background field expanded actions. An algorithm for computing the higher loop diagrams will be given.

\section{IIa. The Two Derivative Chiral Lagrangian}

We will first consider the lowest order ungauged chiral lagrangian where the method will be explained in detail and then the gauged versions will be discussed. 
In each case, a compact form for the fluctuations to all orders can be obtained. (See Eq. (2.15) below.)

The two derivative chiral lagrangian, not including external fields can be written as (we consider chiral $S U(3)$ unless otherwise stated)

$$
\mathscr{L}^{(2)}=\mathscr{L}_{\text {inv }}^{(2)}+\mathscr{L}_{M}^{(2)} .
$$

$\mathscr{L}_{\text {inv }}^{(2)}$ is the invariant part of the lagrangian and is [1]

$$
\mathscr{L}_{\mathrm{inv}}^{(2)}=\frac{f^{2}}{4}\left\langle\partial_{\mu} U^{\dagger} \partial^{\mu} U\right\rangle=-\frac{f^{2}}{4}\left\langle L_{\mu} L^{\mu}\right\rangle,
$$

where $L_{\mu}$ are the group currents;

$$
L_{\mu}=U^{-1} \partial_{\mu} U=U^{\dagger} \partial_{\mu} U
$$

and

$$
\begin{aligned}
\mathscr{L}_{M}^{(2)} & =\frac{f^{2}}{4}\left\langle\chi U^{\dagger}+\chi^{\dagger} U\right\rangle \\
\chi & =2 B_{0} M .
\end{aligned}
$$

$M=\operatorname{diag}\left(m_{u}, m_{d}, m_{s}\right)$ is the quark mass matrix. Throughout this paper we will use the notation $\operatorname{Tr} A \equiv\langle A\rangle$. Here, as usual, $f$ is the pion decay constant in the chiral limit and $B_{0}$ is related to the condensate; $\langle 0|\bar{u} u| 0\rangle=-f^{2} B_{0}\left(1+O\left(m_{\text {quark }}\right)\right.$ [1]. $U(x)$ is a $3 \times 3$ matrix incorporating the light meson fields, which can be parametrized as:

$$
U(x)=e^{i \phi(x)} \frac{}{f},
$$

$\phi(x)=\phi^{a} \lambda^{a}$ ( $\lambda^{a}$ are the Gellmann matrices) is the pseudo scalar meson octet. Under $S U(3)_{L} \times S U(3)_{R}$ transformations $U$ transforms as $V_{R} U(x) V_{L}^{\dagger}$, where $V_{L(R)}$ belong to $S U(3)_{L(R)}$. In order to evaluate the loop graphs generated by $\mathscr{L}^{(2)}$, we consider the neighborhood of the solution $\bar{U}(x)$ to the classical equations of motion. We will formulate the background field expansion using curves on the group manifold which pass through both the background field $\bar{U}(x)$ and the background field with the fluctuations, $U(x)$. For this purpose we introduce a one-parameter family of group elements $U(x, t)$ such that

$$
\begin{aligned}
U(x, 0) & =\bar{U}(x) \\
U(x, 1) & =U(x)=u\left(e^{-i \xi}\right) u \\
\bar{U}(x) & =u^{2} .
\end{aligned}
$$


$\xi(x)$ is the fluctuation field and is a traceless hermitian matrix; $\xi=\xi^{a} \lambda^{a} ; u(x)$ are elements of the coset space $S U(3)_{L} \times S U(3)_{R} / S U(3)_{V}$ which transform under $S U(3)_{L} \times S U(3)_{R}$ as

$$
u \rightarrow V_{R} u h^{\dagger}=h u V_{L}^{+},
$$

where $h \in S U(3)_{V}$. We next introduce the $t$-dependent action. (We consider $\mathscr{L}_{\text {inv }}^{(2)}$ only first)

$$
\begin{aligned}
S(t) & =\int d^{4} x t x \mathscr{L}^{(2)}(t) \\
& =\frac{f^{2}}{4} \int d^{4} x\left\langle\partial_{\mu} U^{+}(x, t) \partial^{\mu} U(x, t)\right\rangle \\
& =-\frac{f^{2}}{4} \int d^{4} x\left\langle L_{\mu}(t) L^{\mu}(t)\right\rangle,
\end{aligned}
$$

where $L_{\mu}(t)=U^{-1}(x, t) \partial_{\mu} U(x, t)$. The action incorporating the fluctuations to all orders may be written as

$$
S(1)-S(0)=\int_{0}^{1} d t \frac{\partial}{\partial t} S(t)
$$

Our task now is to evaluate the RHS of Eq. (2.6). In order to do this, we first obtain the geodesic equation satisfied by the currents $L_{\mu}(t)$ :

$$
\begin{aligned}
\frac{d L_{\mu}(t)}{d t} & =\frac{d}{d t}\left(U_{t}^{-1} \partial_{\mu} U_{t}\right) \\
& =\partial_{\mu}\left(U_{t}^{-1} \dot{U}_{t}\right)+\left[U_{t}^{-1} \partial_{\mu} U_{t}, U_{t}^{-1} \dot{U}_{t}\right] \\
& =\partial_{\mu} A+\left[L_{\mu}(t), A\right],
\end{aligned}
$$

where we have introduced the notations, $U_{t}=U(x, t), \dot{U}_{t}=(\partial / \partial t) U_{t}$, and $U_{t}^{-1} \dot{U}_{t}=\Lambda$. (This geodesic equation plays a crucial role since the various terms in the chiral lagrangian can be always expressed in terms of $L_{\mu}$.) From (2.7) it immediately follows that

$$
\mathscr{L}(1)-\mathscr{L}(0)=-\frac{f^{2}}{2} \int_{0}^{1} d t\left\langle\partial_{\mu} A L_{\mu}(t)\right\rangle .
$$

It is to be noted that $\left.(\partial S(t) / \partial t)\right|_{t=0}$ vanishes by the equations of motion. To proceed further, we specialize to the case of interest, i.e., the nonlinear sigma models on group manifolds for which we may introduce the exponential parametrization for $U(x, t)$, namely,

$$
U(x, t)=u e^{-i \xi t} u .
$$


In this parametrization, $A=U_{t}^{-1} \dot{U}_{t}=u^{-1}(-i \xi) u=\bar{U}^{-1} u(-i \xi) u$. In order to simplify the subsequent expressions we introduce the following anti-hermitian matrices [1]

$$
\begin{aligned}
\Delta_{\mu} & =\frac{1}{2}\left(u^{-1} \partial_{\mu} u+\partial_{\mu} u u^{-1}\right) \\
& =\frac{1}{2} u^{-1} \partial_{\mu} \bar{U} u^{-1}=-\frac{1}{2} u \partial_{\mu} \bar{U}^{\dagger} u \\
& =\frac{1}{2} u L_{\mu} u^{\dagger} \\
\Gamma_{\mu} & =\frac{1}{2}\left(u^{-1} \partial_{\mu} u-\partial_{\mu} u u^{-1}\right) .
\end{aligned}
$$

$\Gamma_{\mu}$ transforms like a connection under $S U(3)_{L} \times S U(3)_{R}$ and $\Delta_{\mu}$ transforms covariantly as is explicitly discussed in the next section (IIIc). Thus it is convenient to introduce the covariant derivative of $\xi[1]$,

$$
d_{\mu} \xi=\partial_{\mu} \xi+\left[\Gamma_{\mu}, \xi\right]
$$

In terms of (2.9) and (2.10)

$$
\partial_{\mu}(u \xi u)=u\left\{d_{\mu} \xi+\left\{\Delta_{\mu}, \xi\right\}\right\} u .
$$

Returning next to (2.8) we may rewrite it as

$$
\begin{aligned}
\mathscr{L}(1)-\mathscr{L}(0)= & \frac{f^{2}}{2} i \int_{0}^{1} d t\left\{\left\langle u \xi u U_{t}^{-1} \partial_{\mu} U_{t} \partial_{\mu} \bar{U}^{-1}\right\rangle\right. \\
& \left.+\left\langle\partial_{\mu}(u \xi u) U_{t}^{-1} \partial_{\mu} U_{t} \bar{U}^{-1}\right\rangle\right\} .
\end{aligned}
$$

Next, using

$$
U_{t}^{-1} \partial_{\mu} U_{t}=u^{-1}\left\{e^{i \xi t}\left(\partial_{\mu}+\Delta_{\mu}+\Gamma_{\mu}\right) e^{-i \zeta^{t} t}+\Delta_{\mu}-\Gamma_{\mu}\right\} u,
$$

we can write $(2.12)$ as

$$
\begin{aligned}
\mathscr{L}(1)-\mathscr{L}(0)= & \frac{f^{2}}{2} i \int_{0}^{1} d t\left\{\left\langle\left(d_{\mu} \xi-\left[\Delta_{\mu}, \xi\right]\right)\left(e^{i \xi s}\left(\partial_{\mu}+\Delta_{\mu}+\Gamma_{\mu}\right) e^{-i \xi s}\right)\right\rangle\right. \\
& \left.+\left\langle\left(d_{\mu} \xi-\left[\Delta_{\mu}, \xi\right]\right)\left(\Delta_{\mu}-\Gamma_{\mu}\right)\right\rangle\right\} .
\end{aligned}
$$

This expression may be further simplified using the parametrization:

$$
e^{i \xi s}\left(\partial_{\mu}+\Delta_{\mu}+\Gamma_{\mu}\right) e^{-i \xi s}=-i \int_{0}^{t} d s e^{i \xi s}\left(d_{\mu} \xi+\left[\Delta_{\mu}, \xi\right]\right) e^{-i \xi \xi}+\left(\Delta_{\mu}+\Gamma_{\mu}\right) .
$$

Thus, Eq. (2.13) becomes

$$
\begin{aligned}
\mathscr{L}(1)-\mathscr{L}(0)= & \frac{f^{2}}{2} \int_{0}^{1} d t \int_{0}^{l} d s\left\langle\left\{ e^{-i \xi s}\left(d^{\mu} \xi-\left[\Delta^{\mu}, \xi\right]\right) e^{i \xi s}\right.\right. \\
& \left.\left.\times\left(d_{\mu} \xi+\left[\Delta^{\mu}, \xi\right]\right)\right\}\right\rangle+f^{2} i\left\langle\Delta_{\mu} d_{\mu} \xi\right\rangle .
\end{aligned}
$$


For future reference we note that the above may be written in an alternate convenient form, using

$$
\begin{aligned}
d_{\mu} \xi-\left[\Delta_{\mu} \xi\right] & =\partial_{\mu} \xi-\left[\partial_{\mu} u u^{-1}, \xi\right] \\
& =u \partial_{\mu}\left(u^{-1} \xi u\right) u^{-1} \\
d_{\mu} \xi+\left[\Delta_{\mu} \xi\right] & =\partial_{\mu} \xi+\left[u^{-1} \partial_{\mu} U, \xi\right] \\
& =u\left(\partial_{\mu}\left(u^{-1} \xi u\right)+\left[L_{\mu}, u^{-1} \xi u\right]\right) u^{-1} .
\end{aligned}
$$

We may write $(2.15 \mathrm{a})$ as

$$
\begin{aligned}
\mathscr{L}(1)-\mathscr{L}(0)= & \frac{f^{2}}{2} \int_{0}^{1} d t \int_{0}^{t} d s\left\langle e^{-i \xi s} u \partial^{\mu}\left(u^{-1} \xi u\right) u^{-1} e^{i \xi s}\right. \\
& \left.\times u\left(\partial_{\mu}\left(u^{-1} \xi u\right)+\left[L_{\mu}, u^{-1} \xi u\right]\right) u^{-1}\right\rangle \\
& + \text { term linear in } \xi \\
= & -\frac{f^{2}}{2} \int_{0}^{1} d t \int_{0}^{l} d s\left\langle e^{A s} \partial^{\mu} A e^{-A s} \tilde{D}_{\mu} A\right\rangle \\
& + \text { term linear in } \xi,
\end{aligned}
$$

where

$$
\widetilde{D}_{\mu} A=\partial_{\mu} A+\left[L_{\mu}, A\right] .
$$

The term linear in $\xi$ is seen to vanish upon using the equation of motion for the background fields (for the massless case: $d_{\mu} \Delta^{\mu}=0$ ). Equation $(2.15 \mathrm{~b})$ reduces to the result of Ref. [1] for the case of the quadratic fluctuations and the higher order fluctuations may be easily read off. Below we list some of these:

$$
\begin{gathered}
\text { Cubic: } \frac{f^{2}}{6} i\left\langle\left[\Delta^{\mu}, \xi\right]\left[d_{\mu} \xi, \xi\right]\right\rangle \\
\text { Quartic: } \frac{f^{2}}{24} i\left\{\left\langle\left[d_{\mu} \xi, \xi\right]^{2}-\left[\Delta_{\mu}, \xi\right]^{2}\right\rangle\right\} \\
\text { Fifth order: } \frac{f^{2}}{20}\left\{\left\langle\left[\left[\left[\Delta^{\mu}, \xi\right], \xi\right], \xi\right]\left[d_{\mu} \xi, \xi\right]\right\rangle\right\} .
\end{gathered}
$$

We conclude this discussion of the chiral lagrangian without external fields by writing down the expansion of $\mathscr{L}_{M}^{2}$ which is straightforward to obtain using the above techniques:

$$
\mathscr{L}_{M}^{(2)}(1)-\mathscr{L}_{M}^{(2)}(0)<-\frac{i f^{2}}{4} \int_{0}^{1} d t\left\langle\xi\left(u \chi^{\dagger} u e^{-i \xi t}-e^{+i \xi t} u^{\dagger} \chi u^{\dagger}\right)\right\rangle .
$$

Of course, here $\bar{U}=u^{2}$ is the classical equation of motion of the complete lagrangian (2.1); i.e.,

$$
d_{\mu} \Delta^{\mu}-\frac{1}{2} M^{-}+\frac{1}{6}\left\langle M^{-}\right\rangle=0
$$


with

$$
M^{-}=B_{0}\left(u^{\dagger} M u^{\dagger}-u M u\right) .
$$

We note that when (2.17) is expanded in the fluctuations, the background fields appear either as $M^{-}$or $M^{+}=B_{0}\left(u^{\dagger} M u^{\dagger}+u M u\right)$. Terms odd in $\xi$ appear with $M^{-}$ and those even in $\xi$ with $M^{+}$.

In order to consider the low encrgy Green's functions of the vector, axial-vector, scalar and pseudoscalar quark currents in QCD, one has to consider the chiral lagrangian coupled to external vector fields $v_{\mu}$, axial-vector fields $a_{\mu}$, scalars $s$, and pseudoscalars $p[1]$. The lowest order chiral lagrangian incorporating these may be written in the form (2.1) with

$$
\begin{aligned}
& \mathscr{L}_{\mathrm{inv}}^{(2)}=\frac{f^{2}}{4}\left\langle D_{\mu} U D^{\mu} U^{\dagger}\right\rangle \\
& \mathscr{L}_{M}^{(2)}=\frac{f^{2}}{4}\left\langle\chi U^{\dagger}+\chi^{\dagger} U\right\rangle,
\end{aligned}
$$

where

$$
\begin{aligned}
D_{\mu} U & =\partial_{\mu} U-i F_{\mu}^{R} U-i U F_{\mu}^{L} \\
F_{\mu}^{L, R} & =v_{\mu} \mp a_{\mu} \\
\chi & =2 B_{0}(s+i p) .
\end{aligned}
$$

The external fields transform under $S U(3)_{L} \times S U(3)_{R}$ in the following manner:

$$
\begin{aligned}
F_{\mu}^{L, R} & \rightarrow V_{L, R}\left(F_{\mu}^{L, R}\right) V_{L, R}^{\dagger}+i V_{L, R} \partial_{\mu} V_{L, R}^{\dagger} \\
s+i p & \rightarrow V_{R}(s+i p) V_{L}^{\dagger} .
\end{aligned}
$$

The background field expansion of the lagrangian (2.19) can be performed in exactly an analogous manner as discussed earlier. The result for the fluctuations to all orders has the same form as Eq. (2.15) with the following replacements for $\Gamma_{\mu}$ and $\Delta_{\mu}$ :

$$
\begin{aligned}
& \Gamma_{\mu}=\frac{1}{2}\left(u^{-1} \partial_{\mu} u-\partial_{\mu} u u^{-1}\right)-\frac{1}{2} i u^{-1} F_{\mu}^{R} u-\frac{1}{2} i u F_{\mu}^{L} u^{-1} \\
& \Delta_{\mu}=\frac{1}{2} u^{-1} D_{\mu} \bar{U} u^{-1}=-\frac{1}{2} u D_{\mu} \bar{U}^{\dagger} u .
\end{aligned}
$$

$\bar{U}$ is the solution of the classical equations of motion obtained from (2.19) which has the same form as $(2.18)$ with the appropriate replacements $(2.19 \mathrm{~b})$.

\section{IIb. The Wess-Zumino Lagrangian}

In this section the background field expansion for the Wess-Zumino lagrangian, appropriate for the description of anomalous processes in chiral perturbation 
theory will be discussed. This lagrangian is $\mathcal{O}\left(p^{4}\right)$ and was first obtained by Wess and Zumino [2]. More recently, Witten [3] has obtained a compact form for the action and the first terms are shown to be expressed in terms of the field $U$ as an integral over five dimensions. Expressed in terms of the fields $\phi$, the action reduces to a four-dimensional form modulo topological effects. Thus, the essential feature of such terms is that though the action is not a total divergence in terms of the $U$ fields, it can be expressed as a total divergence in terms of the fields $\phi$. In order to consider corrections in chiral perturbation theory to anomalous processes like $\pi^{0} \rightarrow \gamma \gamma, \eta \rightarrow \pi^{+} \pi^{-} \gamma$, etc., it is necessary to develop a background field expansion of this lagrangian and we discuss a procedure below where all the symmetries of the original lagrangian may be preserved. This procedure, which is based on the strategy outlined in the previous subsection, makes clear the fact that to all orders, the fluctuations can be expressed in a compact form in four dimensions in terms of the fields $U$. Apart from the four-dimensional case which is of physical interest in discussing meson interactions, we will also discuss the two-dimensional Wess-Zumino model, where our expansion has many simplifications over the methods previously employed $[8,9]$. In Section III we will discuss the important issue of the non-renormalization of the coeflicient of the Wess-Zumino term in general. As before, we will first discuss the models without external fields and then these fields will be introduced.

In both four and two dimensions, we will use Witten's form for the Wess-Zumino lagrangian. We first consider the four-dimensional case and later the two-dimensional model will be discussed,

$$
\begin{aligned}
S_{\mathrm{WZ}} & =\int d^{5} x \mathscr{L}_{\mathrm{WZ}} \\
& =\frac{-i N_{c}}{240 \pi^{2}} \int_{\partial D=M_{4}} \varepsilon^{\alpha \beta \gamma \delta \rho}\left\langle L_{\alpha} L_{\beta} L_{\gamma} L_{\sigma} L_{\rho}\right\rangle d^{5} x \\
& =\frac{-i N_{c}}{240 \pi^{2}} \int_{\partial D=M_{4}}\left\langle L^{5}\right\rangle,
\end{aligned}
$$

where $L=L_{\mu} d x^{\mu}=U^{-1} \partial_{\mu} U d x^{\mu}$ and we are using the language of differential forms which is very well suited for this situation. In Eq. (2.21) $N_{c}$ is the number of colors and the integral is over a five-dimensional surface $D$, whose boundary is four-dimensional space-time. As in the previous section, we consider the one parameter extensions of the chiral field $U(x, t) \equiv U_{t}$ and construct the lagrangian incorporating the fluctuations. (We omit for the moment an overall factor of $-i N_{c} / 240 \pi^{2}$ in $\left.\mathscr{L}.\right)$

$$
\begin{aligned}
\mathscr{L}_{\mathrm{WZ}}(1)-\mathscr{L}_{\mathrm{WZ}}(0) & =\int_{0}^{1} \frac{\partial}{\partial t}\left\langle L_{t}^{5}\right\rangle . \\
L_{t} & =U_{t}^{-1} d U_{t} .
\end{aligned}
$$


It is straightforward to show using Eq. (2.7) that the RHS of (2.22) is a total divergence to all orders in the fluctuation:

$$
\begin{aligned}
\mathscr{L}_{\mathrm{WZ}}(1)-\mathscr{L}_{\mathrm{WZ}}(0) & =5 \int_{0}^{1} d t\left\langle\left(\frac{\partial}{\partial t} L_{t}\right) L_{t}^{4}\right\rangle \\
& =5 \int_{0}^{1} d t d\left\langle A L_{t}^{4}\right\rangle
\end{aligned}
$$

Thus,

$$
S_{\mathrm{W}}(1)-S_{\mathrm{WZ}}(0)=\frac{-i N_{c}}{48 \pi^{2}} \int_{0}^{1} d t \int_{M_{4}}\left\langle\Lambda L_{t}^{4}\right\rangle d^{4} x .
$$

Using the identity $d L=-L^{2}$, the above expression may be written in many equivalent forms:

$$
\begin{aligned}
S_{\mathrm{WZ}}(1)-S_{\mathrm{WZ}}(0) & =\frac{-i N_{c}}{48 \pi^{2}} \int_{0}^{1} d t \int_{M_{4}}\left\langle\Lambda d L_{t} d L_{t}\right\rangle \\
& =\frac{-i N_{c}}{48 \pi^{2}} \int_{0}^{1} d t \int_{M_{4}}\left\langle d \Lambda L_{t} d L_{t}\right\rangle .
\end{aligned}
$$

Any one of these forms logether with Eqs. (2.12a) and (2.14); i.e.,

$$
L_{t}=u^{-1}\left\{-i \int_{0}^{t} d s e^{i \xi s}(\bar{d} \xi+[\Delta, \xi]) e^{-i \xi s}+2 \Delta\right\} u,
$$

where $\bar{d} \xi=d \xi+[\Gamma, \xi]$ may be used to write a compact expression for (2.23). One such form is

$$
\begin{aligned}
S_{\mathrm{WZ}}(1) & -S_{\mathrm{WZ}}(0) \\
= & \frac{-i N_{c}}{48 \pi^{2}} \int_{0}^{1} d t \int_{M_{4}}\left\langle-i \xi\left(-i \int_{0}^{t} d s e^{i \xi s}(\bar{d} \xi+[\Delta, \xi]) e^{-i \xi s}+2 \Delta\right)^{4}\right\rangle \\
= & \frac{-i N_{c}}{48 \pi^{2}} \int_{0}^{1} d t \int d^{4} x \varepsilon^{\mu v \rho \sigma}\left\langle-i \xi 2-i \int_{0}^{t} d s e^{i \xi s} D_{+\mu} \xi e^{-i \xi s}+2 \Delta_{\mu}\right) \\
& \times\left(-i \int_{0}^{t} d s e^{i \xi s} D_{+\nu} \xi e^{-i \xi s}+2 \Delta_{v}\right) \\
& \times\left(-i \int_{0}^{t} d s e^{i \xi s} D_{+\rho} \xi e^{-i \xi s}+2 \Delta_{\rho}\right) \\
& \left.\times\left(-i \int_{0}^{t} d s e^{i \xi s} D_{+\sigma} \xi e^{-i \xi s}+2 \Delta_{\sigma}\right)\right\rangle,
\end{aligned}
$$

where

$$
D_{+\mu} \xi=d_{\mu} \xi+\left[\Delta_{\mu}, \xi\right]
$$


It is worth reemphasizing that this lagrangian incorporating the fluctuations can be written in compact form in four dimensions to all orders in the fluctuation. For the second-order fluctuations, this fact was noted in $[6,7]$.

The fluctuations to any order can be readily obtained from this and some of these will be tabulated after a discussion of the Wess-Zumino lagrangian in the presence of external fields. Before this is done we will briefly discuss the two-dimensional version in order to illustrate the simplicity of our techniques. The two-dimensional Wess-Zumino model for the group $S U(N)$ is given by the action

$$
\left.S\right|_{2 D}=\frac{f^{2}}{4} \int d^{2} x\left\langle\partial_{\mu} U^{-1} \partial^{\mu} U\right\rangle+\kappa \frac{f^{2}}{6} \int_{\partial D=M_{2}}\left\langle L^{3}\right\rangle .
$$

For $\kappa^{2}=1$, the model is known to be conformally invariant [10], and we will show in Section IV, that for this case, the WZ term is unrenormalized. The first term is of the same form as in Section Ia and therefore we will focus on the second term alone. Proceeding as before, it is straightforward to see that in this case:

$$
\begin{aligned}
S_{\mathrm{WZ}}(1)-\left.S_{\mathrm{WZ}}(0)\right|_{2 D} & =\kappa \frac{f^{2}}{2} \int_{0}^{1} d t \int_{M_{2}}\left\langle A L_{t}^{2}\right\rangle d^{2} x \\
& =\kappa \frac{f^{2}}{2} \int_{0}^{1} d t \int_{M_{2}}\left\langle d \Lambda L_{t}\right\rangle .
\end{aligned}
$$

This is very similar to the form of the first term of Eq. (2.8) and we may write for the fluctuations obtained from (2.26), using (2.15),

$$
\begin{aligned}
S_{\mathrm{WZ}}(1)-\left.S_{\mathrm{WZ}}(0)\right|_{2 D}= & \frac{f^{2}}{2}\left(g_{\mu v}-\kappa \varepsilon_{\mu v}\right) \int_{0}^{1} d t \int_{0}^{t} d s \int d^{2} x \\
& \times\left\{\left\langle e^{-i \xi_{s}}\left(d_{\mu} \xi-\left[\Delta_{\mu}, \xi\right]\right) e^{i \xi s}\left(d_{v} \xi+\left[\Delta_{v}, \xi\right]\right)\right\rangle\right\} \\
& -f^{2} i \int\left(g_{\mu v}-\kappa \varepsilon_{\mu v}\right)\left\langle\xi\left(d_{\mu} \Delta_{v}-\left[\Delta_{\mu}, \Delta_{v}\right]\right)\right\rangle d^{2} x .
\end{aligned}
$$

The term that is linear in $\xi$ in (2.27) is seen to vanish upon using the equations of motion for (2.26), i.e.,

$$
d_{\mu} \Delta^{\mu}+\kappa \varepsilon^{\mu v}\left[\Delta_{\mu}, \Delta_{v}\right]=0 .
$$

Before proceeding with a discussion of the inclusion of external gauge fields we would like to indicate some alternate forms for Eqs. (2.25) which are convenient for explicit calculations. Consider first Eq. (2.25b). The covariant derivative $D_{+\mu} \xi$ may be written as

$$
D_{+\mu} \xi=\partial_{\mu} \xi+\left[u^{-1} \partial_{\mu} u, \xi\right]=u^{-1} \partial_{\mu}\left(u \xi u^{-1}\right) u .
$$

Using this, $(2.25 \mathrm{~b})$ may also be written as 


$$
\begin{aligned}
S_{\mathrm{WZ}}(1)-S_{\mathrm{WZ}}(0)= & \frac{-i N_{c}}{48 \pi^{2}} \int_{0}^{1} d t \int d^{4} x \varepsilon^{u v \rho \sigma} \\
& \times\left\langle-i \xi^{\prime}\left(-i \int_{0}^{t} d s e^{i \xi^{\prime} s} \partial_{\mu} \xi^{\prime} e^{-i \xi^{\prime} s}+R_{\mu}\right)\right. \\
& \times\left(-i \int_{0}^{t} d s e^{i \xi^{\prime} s} \partial_{\nu} \xi^{\prime} e^{-i \xi^{\prime} s}+R_{v}\right) \\
& \times\left(-i \int_{0}^{t} d s e^{i \xi^{\prime} s} \partial_{\rho} \xi^{\prime} e^{-i \xi^{\prime} s}+R_{\rho}\right) \\
& \left.\times\left(-i \int_{0}^{t} d s e^{i \xi^{\prime} s} \partial_{\sigma} \xi^{\prime} e^{-i \xi^{\prime} s}+R_{\sigma}\right)\right\rangle
\end{aligned}
$$

with $\xi^{\prime}=u \xi u^{-1}$ and $R_{\mu}=\partial_{\mu} U U^{-1}$.

The alternate form for (2.27) follows from Eq. (2.15b),

$$
\begin{aligned}
S_{\mathrm{WZ}}(1)-\left.S_{\mathrm{WZ}}(0)\right|_{2 D}= & \frac{f^{2}}{2}\left(g^{\mu v}-\kappa \varepsilon^{\mu v}\right) \int_{0}^{1} d t \int_{0}^{t} d s \int d^{2} x \\
& \times\left\{\left\langle e^{-i \xi_{s}} \partial_{\mu} \xi e^{i \xi_{s}}\left(\partial_{v} \xi+2\left[u^{-1} \Delta_{v} u, \xi\right]\right)\right\rangle\right\},
\end{aligned}
$$

where $\tilde{\xi}=u^{-1} \xi u$ and we are assuming that the background field equations of motion are satisfied.

We will next consider the Wess-Zumino effective lagrangian in four dimensions in the presence of external vector and axial vector gauge fields. In the context of chiral perturbation theory, this is necessary in order to consider the correlation functions of the various quark currents in a low energy expansion. The form of the Wcss-Zumino functional in four dimensions in the presence of gauge fields $F_{\mu}^{L}, F_{\mu}^{R}$ has been obtained by a number of authors, and we will use the one given in Ref. [4] with a straightforward adaptation to our conventions and notations:

$$
\begin{aligned}
S_{\mathrm{WZ}}\left(F^{L}, F^{R}, U\right)= & \frac{-i N_{c}}{240 \pi^{2}} \int_{\partial D=M_{4}}\left\langle\left(U^{-1} d U\right)^{5}\right\rangle \\
& \times \frac{-i N_{c}}{48 \pi^{2}} \int_{M_{4}}\left\langle\left\{-i F^{R}\left(d U U^{-1}\right)^{3}-\right.\right.\text { P.C. } \\
& +\left(-F^{R} d F^{R}-d F^{R} F^{R}+i\left(F^{R}\right)^{3}\right)\left(-i U F^{L} U^{-1}+U d U^{-1}\right)-\text { P.C. } \\
& -\left(d U U^{-1} d F^{R} U F^{L} U^{-1}-\text { P.C. }\right) \\
& -\frac{1}{2}\left(F^{R} d U U^{-1} F^{R} d u U^{-1}-\text { P.C. }\right) \\
& -\left(U F^{L} U^{-1} F^{R} d U U^{-1} d U U^{-1}-\text { P.C. }\right) \\
& -i\left(F^{L} U^{-1} d U F^{L} U^{-1} F^{R} U-\text { P.C. }\right) \\
& \left.\left.-\frac{1}{2} F^{L} U^{-1} F^{R} U F^{L} U^{-1} F^{R} U\right\}\right\rangle .
\end{aligned}
$$

(Here, P.C. stands for parity conjugate: $F^{L} \leftrightarrow F^{R}, U \leftrightarrow U^{-1}$.) 
The action which includes, the fluctuations to all orders can be obtained by the same techniques as before. After straightforward algebra, we obtain

$$
\begin{aligned}
S_{\mathrm{wZ}}( & \left.U(x, t=1), F_{L}^{\mu}, F_{R}^{\mu}\right)-S_{\mathrm{wZ}}\left(U(x, t=0), F_{L}^{\mu}, F_{R}^{\mu}\right) \\
= & \frac{-i N_{c}}{48 \pi^{2}} \int_{M_{4}} \int_{0}^{1} d t\left\langle\left\{U _ { t } ^ { - 1 } \dot { U } _ { t } \left\{\left(U_{t}^{-1} D U_{t}\right)^{4}+i\left(U_{t}^{-1} D U_{t}\right)^{2}\right.\right.\right. \\
& \times\left(F_{L}^{(2)}+U_{t}^{-1} F_{R}^{(2)} U_{t}\right)+i\left(F_{L}^{(2)}+U_{t}^{-1} F_{R}^{(2)} U_{t}\right)\left(U_{t}^{-1} D U_{t}\right)^{2} \\
& \times i U_{t}^{-1} D U_{t}\left(F_{L}^{(2)}+U_{t}^{-1} F_{R}^{(2)} U_{t}\right) U_{t}^{-1} D U_{t} \\
& +2\left(\left(F_{L}^{(2)}\right)^{2}+\left(U_{t}^{-1} F_{R}^{(2)} U_{t}\right)^{2}\right) \\
& \left.\left.\left.+F_{L}^{(2)} U_{t}^{-1} F_{R}^{(2)} U_{t}+U_{t}^{-1} F_{R}^{(2)} U_{t} F_{L}^{(2)}\right\}\right\}\right\rangle .
\end{aligned}
$$

In the above, $D U_{t}$ is the covariant derivative

$$
D U_{t}=d U_{t}-i F_{R} U_{t}+i U_{t} F_{L}
$$

and $F_{L, R}^{(2)}$ are the field strength two-forms

$$
F_{L, R}^{(2)}=\frac{1}{2} F_{\mu \nu}^{L, R} d x^{\mu} d x^{\nu}=d F_{L, R}-i F_{L, R}^{2}
$$

or

$$
F_{\mu v}^{L, R}=\partial_{\mu} F_{v}^{L, R}-\partial_{v} F_{\mu}^{L, R}-i\left[F_{\mu}^{L, R}, F_{v}^{L, R}\right]
$$

The above may be further rewritten using the parametrizations $U_{t}=u e^{-i \xi t} u$ and Eq. (2.12a). We first introduce the quantities

$$
\begin{aligned}
F^{ \pm} & =i\left(u F_{L}^{(2)} u^{-1} \pm u^{-1} F_{R}^{(2)} u\right) \\
\Gamma^{(2)} & =\frac{1}{2} \Gamma_{\mu v} d x \mu d x^{v}=d \Gamma+\Gamma^{2}
\end{aligned}
$$

or

$$
\Gamma_{\mu v}=d_{\mu} \Gamma_{v}-\partial_{v} \Gamma_{\mu}+\left[\Gamma_{\mu}, \Gamma_{v}\right] .
$$

The $\Gamma$ are defined in Eq. $(2.9 \mathrm{~b})$. It is easy to check that

$$
\Gamma^{(2)}=-\Delta^{2}-\frac{1}{2} F^{+},
$$

i.e.,

$$
\Gamma_{\mu \nu}=-\left[\Delta_{\mu}, \Delta_{v}\right]-\frac{1}{2} F_{\mu v}^{+}
$$

$\Delta$ is also defined in (2.9b). Using these quantities, the various terms in Eq. (2.29) may be written as 


$$
\begin{aligned}
& \frac{-i N_{c}}{48 \pi^{2}} \int_{M_{4}} \int_{0}^{l} d t\left\langle U_{t}^{-1} \dot{U}_{t}\left(U_{t}^{-1} D U_{t}\right)^{4}\right\rangle \\
& =\frac{-i N_{c}}{48 \pi^{2}} \int_{M_{4}} \int_{0}^{1} d t\left\langle(-i \xi)\left\{-i \int_{0}^{t} d s e^{i \xi s}(\bar{d} \xi+[\Delta, \xi]) e^{-i \xi s}+2 \Delta\right\}^{4}\right\rangle \\
& \frac{-i N_{c}}{48 \pi^{2}} \int_{M_{4}} \int_{0}^{1} d t i\left\langle U_{t}^{-1} \dot{U}_{t}\left(U_{t}^{-1} D U_{t}\right)^{2}\left(F_{L}^{(2)}+U_{t}^{-1} F_{R}^{(2)} U_{t}\right)\right\rangle \\
& =\frac{-i N_{c}}{48 \pi^{2}} \int_{M_{4}} \int_{0}^{1} d t\left\langle(-i \xi)\left\{-i \int_{0}^{t} d s e^{i \xi s}(\bar{d} \xi+[\Delta, \xi]) e^{-i \xi s}+2 \Delta\right\}^{2}\right. \\
& \left.\times\left\{+i \int_{0}^{t} d s e^{i \xi s}\left[\Gamma^{(2)}+\Delta^{2}+\frac{1}{2} F^{-}, \xi\right] e^{-i \xi s}+-2\left(\Gamma^{(2)}+\Delta^{2}\right)\right\}\right\rangle \\
& \frac{-i N_{c}}{48 \pi^{2}} \int_{M_{4}} \int_{0}^{1} d t i\left\langle U_{t}^{-1} \dot{U}_{t}\left(F_{L}^{(2)}+U_{t}^{-1} F_{R}^{(2)} U_{t}\right)\left(U_{t}^{-1} D U_{t}\right)^{2}\right\rangle \\
& =\frac{-i N_{c}}{48 \pi^{2}} \int_{M_{4}} \int_{0}^{1} d t\langle(-i \xi) \\
& \times\left\{i \int_{0}^{t} d s e^{i \xi_{s}}\left[\Gamma^{(2)}+\Delta^{2}+\frac{1}{2} F^{-}, \xi\right] e^{-i \xi s}+\left(\Gamma^{(2)}+\Delta^{2}\right)\right\} \\
& \left.\times\left\{-i \int_{0}^{t} d s e^{i \xi s}(\bar{d} \xi+[\Delta, \xi]) e^{-i \xi \xi s}+2 \Delta\right\}^{2}\right) \\
& \frac{-i N_{c}}{48 \pi^{2}} \int_{M_{4}} \int_{0}^{1} d t i\left\langle U_{t}^{-1} \dot{U}_{t} U_{t}^{-1} D U_{t}\left(F_{L}^{(2)}+U_{t}^{-1} F_{R}^{(2)} U_{t}\right) U_{t}^{-1} D U_{t}\right\rangle \\
& =\frac{-i N_{c}}{48 \pi^{2}} \int_{M_{4}} \int_{0}^{1} d t\left\langle(-i \xi)\left\{-i \int_{0}^{t} d s e^{i \xi s}(d \xi+[\Delta, \xi]) e^{-i \xi s}+2 \Delta\right\}\right. \\
& \times\left\{i \int_{0}^{t} d s e^{i \xi s}\left[\Gamma^{(2)}+\Delta^{2}+\frac{1}{2} F^{-}, \xi\right] e^{-i \xi s}+\left(\Gamma^{(2)}+\Delta^{2}\right)\right\} \\
& \left.\times\left\{-i \int_{0}^{t} d s e^{i \xi s}(\bar{d} \xi+[\Delta, \xi]) e^{-i \xi s}+2 \Delta\right\}\right) \\
& \frac{-i N_{c}}{48 \pi^{2}} \int_{M_{4}} \int_{0}^{1} d t\left\langle U_{t}^{-1} \dot{U}_{t} 2\left(\left(F_{L}^{(2)}\right)^{2}+U_{t}^{-1}\left(F_{R}^{(2)}\right)^{2} U_{t}\right)\right\rangle \\
& =\frac{-i N_{c}}{48 \pi^{2}} \int_{M_{4}} \int_{0}^{1} d t 2\left\langle( - i \xi ) \left\{ u\left(F_{L}^{(2)}\right)^{2} u^{-1}+u^{-1}\left(F_{R}^{(2)}\right)^{2} u\right.\right. \\
& \left.\left.+-i \int_{0}^{t} d s e^{i \xi s}\left[u^{-1}\left(F_{R}^{(2)}\right)^{2} u, \xi\right] e^{-i \xi s}\right\}\right\rangle
\end{aligned}
$$




$$
\begin{aligned}
\frac{-i N_{c}}{48 \pi^{2}} \int_{M_{4}} \int_{0}^{1} d t\left\langle U_{t}^{-1} \dot{U}_{t}\left(F_{L}^{(2)} U_{t}^{-1} F_{R}^{(2)} U_{t}+U_{t}^{-1} F_{R}^{(2)} U_{t} F_{L}^{(2)}\right)\right\rangle \\
=\frac{-i N_{c}}{48 \pi^{2}} \int_{M_{4}} \int_{0}^{1} d t\left\langle( - i \xi ) \left\{ u\left(F_{L}^{(2)}\right)^{2} u^{-1} u^{-1}\left(F_{R}^{(2)}\right)^{2} u+u^{-1} F_{R}^{(2)} u u F_{L}^{(2)} u^{-1}\right.\right. \\
\quad \times-i \int_{0}^{t} d s u F_{L}^{(2)} u^{-1} e^{i \xi s}\left[u^{-1} F_{R}^{(2)} u, \xi\right] e^{-i \xi s} \\
\left.\left.\quad \times-i \int_{0}^{t} d s e^{i \xi_{s} s}\left[u^{-1} F_{R}^{(2)} u, \xi\right] e^{-i \xi s} u F_{L}^{(2)} u^{-1}\right\}\right\rangle
\end{aligned}
$$

In the last two expressions $u F_{L}^{(2)} u^{-1}$ and $u^{-1} F_{R}^{(2)} u$ must be replaced by

$$
\begin{aligned}
& u F_{L}^{(2)} u^{-1}=i\left(\Gamma^{(2)}+\Delta^{2}-\frac{1}{2} F^{-}\right) \\
& u^{-1} F_{R}^{(2)} u=i\left(\Gamma^{(2)}+\Delta^{2}+\frac{1}{2} F^{-}\right) .
\end{aligned}
$$

The terms of different orders in the fluctuation can be obtained easily from the above expressions. In the next section we list the quadratic fluctuations. Notice that the basic background field structures appearing in the expansion are $\Gamma, \Delta, \Gamma^{(2)}, F^{-}$.

Once the background field expansions are obtained, we have next to discuss a diagrammatic algorithm for computing the higher order loop diagrams in perturbation theory. In order to do that we make use of the $\Omega[\bar{U}, \ldots]$ functional discussed by deWitt [11] and by deWitt and Grisaru [12]. This functional is defined by

$$
\Omega[\bar{U}, \ldots]=\int[d \xi] \exp \{i\{S(U(x, t=1), \ldots)-S(\bar{U}, \ldots)\}\} .
$$

(The dots denote possible external fields.) $S$ denotes the action and we are assuming that the background field $\bar{U}$ satisfies the classical equations of motion so that terms linear in $\xi$ are absent. This functional generates all diagrams containing at least one loop with external legs amuptated. In Ref. [11] an algorithm to obtain the $S$-matrix elements from $\Omega$ is discussed. The method we follow in generating the diagrams is to express $\Omega[\bar{U}, \ldots]$, using the interaction picture and then the standard Dyson expansion,

$$
\Omega[\bar{U}, \ldots]=\left\langle 0\left|\exp \left\{i \int d^{n} x \mathscr{L}(\bar{U}, \ldots, \xi)\right\}\right| 0\right\rangle,
$$

where

$$
\begin{aligned}
\int d^{n} x \mathscr{L}(\bar{U}, \ldots, \xi)= & S(U(x, t=1), \ldots)-S(\bar{U}, \ldots) \\
& -\frac{f^{2}}{4} \int d^{n} x\left\{\left\langle\partial_{\mu} \xi \partial^{\mu} \xi\right\rangle-\left\langle\xi^{2} 2 B_{0} M\right\rangle\right\} .
\end{aligned}
$$


We may redefine $\xi \rightarrow f \xi$ in order to get the usual normalization for the kinetic term. In the calculation of the vacuum diagrams, the various background field structures $\Delta_{\mu}, \ldots$ may be regarded as external fields together with the other fields $F_{\mu}^{L, R}$ etc., if they exist. We next compute all possible Wick contractions involving the $\xi^{a}$, using

$$
\left\langle 0\left|T\left(\xi^{a}\left(x_{1}\right) \xi^{b}\left(x_{2}\right)\right)\right| 0\right\rangle=i \delta^{a b} \Delta_{F}\left(x_{1}-x_{2}\right),
$$

where $\Delta_{F}\left(x_{1}-x_{2}\right)$ is the massive scalar Feynman propagator. In this way, the various loop effects may be studied in a standard manner. This general procedure is the same as that discussed in the second of Ref. [8].

A glance at Eqs. (2.35), (2.17), (2.15a) tells us immediately that all local counter terms computed using the above described background field method will be chiral symmetric structures constructed out of $\Gamma_{\mu}, \Gamma_{\mu v}, \Delta_{\mu}, M^{ \pm}$, and $F_{\mu \nu}^{-}$, and their covariant derivatives.

\section{ONE-Loop Calculations}

In this section, we will exemplify the general discussion of Section II by means of explicit calculations at the one-loop level. Expanding the total lagrangian $\mathscr{L}=$ $\mathscr{L}_{0}+\mathscr{L}_{\mathrm{wz}}$ (non-linear $\sigma$ model + Wess-Zumino term) Eqs. (2.35), (2.17), and (2.15a) in powers of the quantum fluctuation field $\xi$, only terms quadratic in $\xi$, i.e., $\mathscr{L}^{(2)}$, are needed for one-loop calculations:

$$
\mathscr{L}^{(2)}=\mathscr{L}_{0}^{(2)}+\mathscr{L}_{\mathrm{wZ}}^{(2)},
$$

where

$$
\begin{aligned}
\mathscr{L}_{0}^{(2)}= & \frac{f^{2}}{4}\left\langle\left\{\left(d_{\mu} \xi-\left[\Delta_{\mu}, \xi\right]\right)\left(d_{\mu} \xi+\left[\Delta_{\mu}, \xi\right]\right)-\xi^{2} M^{+}\right\}\right\rangle . \\
\mathscr{L}_{\mathrm{wZ}}^{(2)}= & \frac{-i N_{c}}{48 \pi^{2}} \varepsilon^{\mu \nu \alpha \beta}\left\langle\left\{\left(\xi d_{\mu} \xi-d_{\mu} \xi \xi\right)\left\{\Gamma_{v \alpha}, \Delta_{\beta}\right\}\right.\right. \\
& +\left(\xi \Delta_{\mu} d_{v} \xi-d_{v} \xi \Delta_{\mu} \xi\right) \Gamma_{\alpha \beta} \\
& -2\left(\xi \Delta_{\mu} d_{v} \xi-d_{v} \xi \Delta_{\mu} \xi\right) \Delta_{\alpha} \Delta_{\beta} \\
& -\frac{1}{8} \xi^{2}\left[\Gamma_{\mu v}, F_{\alpha \beta}^{-}\right]+\frac{1}{4} \xi^{2}\left[\Delta_{\mu} \Delta_{v}, F_{\alpha \beta}\right] \\
& \left.\left.-\frac{1}{2} \xi \Delta_{\mu} \xi\left\{F_{v \alpha}^{-}, \Delta_{\beta}\right\}\right\}\right\rangle .
\end{aligned}
$$

The covariant derivative $d_{\mu} \xi$ is defined in (2.10), $M^{-}$in (2.18), $F_{\mu \nu}^{-}$in (2.32) and $M^{+}$is defined after (2.18).

Throughout, we assume $m_{u}=m_{d}$. Feynman rules are derived from $\mathscr{L}^{(2)}$ in the usual way and one-loop Feynman diagrams could be calculated as discussed at the end of Section II. In these diagrams $\xi^{\prime}$ s can only appear in the loops; all others are background fields which appear only as external fields. In the next subsection we 
calculate the divergencies at the one-loop level of the non-anomalous effective lagrangian using this diagrammatic approach. In Subsection III.b we will calculate the one-loop divergencies of the Wess-Zumino lagrangian first without gauge fields, and then the complete result including external vector and axial vector fields will be presented. In III.c we obtain the most general $O\left(p^{6}\right)$ chiral lagrangian in the anomalous sector. This is consistent with parity $P$, charge conjugation $C$, and chiral symmetry.

IIIa. Infinities of the Two-Derivative Chiral Lagrangian

To find the Feynman rules for $\mathscr{L}_{0}^{(2)}$ we set $\xi=\xi^{a} \lambda^{a}$ and obtain

$$
\begin{aligned}
\mathscr{L}_{0}^{(2)}= & f^{2}\left\{\frac{1}{2} \partial_{\mu} \xi^{a} \partial_{\mu} \xi^{a}+\frac{1}{4}\left\langle\left[\lambda^{a}, \lambda^{b}\right] \Gamma_{\mu}\right\rangle\left(\partial_{\mu} \xi^{b} \xi^{a}-\partial_{\mu} \xi^{a} \xi^{b}\right)\right. \\
& +\frac{1}{4} \xi^{a} \xi^{b}\left\langle\left[\lambda^{a}, \Gamma_{\mu}\right]\left[\lambda^{b}, \Gamma_{\mu}\right]\right\rangle \\
& -\frac{1}{4} \xi^{a} \xi^{b}\left\langle\left[\lambda^{a}, \Delta_{\mu}\right]\left[\lambda^{b}, \Delta_{\mu}\right]\right\rangle \\
& \left.-\frac{1}{8} \xi^{a} \xi^{b}\left\langle\left\{\lambda^{a}, \lambda^{b}\right\} M^{+}\right\rangle\right\} .
\end{aligned}
$$
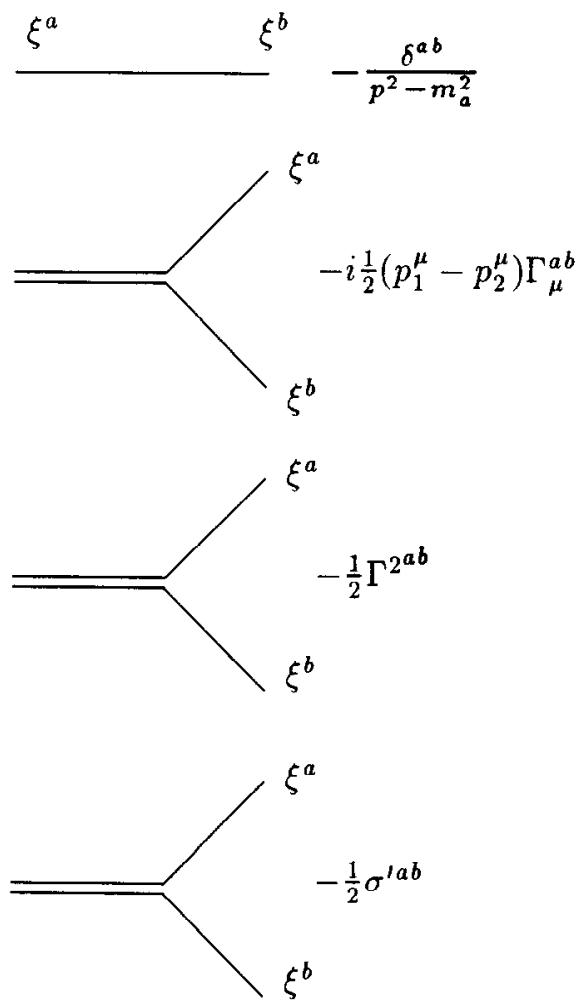

Fig. 1. Feynman rules for the non-anamalous sector. Double lines denote the background fields. 
In addition to the $\xi$ propagator, there are three vertices (Fig. 1). In this, $m_{a}^{2}$ is the mass of $\phi_{a}$ and $\Gamma_{\mu}^{a b},\left(\Gamma^{2}\right)^{a b}$, and $\sigma^{a b}$ are the Fourier transforms of $\Gamma_{\mu}^{a b},\left(\Gamma^{2}\right)^{a b}$, and $\sigma^{\prime a b}$, respectively,

$$
\begin{aligned}
\Gamma_{\mu}^{a b} & =-\frac{1}{2}\left\langle\left[\lambda^{a}, \lambda^{b}\right] \Gamma_{\mu}\right\rangle \\
\sigma^{a b} & =\sigma^{a b}-m_{a}^{2} \delta^{a b} \\
\sigma^{a b} & =\frac{1}{2}\left\langle\left[\lambda^{a}, \Delta_{\mu}\right]\left[\lambda^{b}, \Delta_{\mu}\right]\right\rangle+\frac{1}{4}\left\langle\left\{\lambda^{a}, \lambda^{b}\right\} M^{+}\right\rangle .
\end{aligned}
$$

Our notation is that of Gasser and Leutwyler [1]. The double lines in the vertices denote collectively the background fields contained in $\Gamma_{\mu}, \Gamma^{2}$, or $\sigma$. Using these vertices the one-loop divergencies of the non-anomalous sector can be calculated. In doing so, we have to consider Feynman diagrams up to the fourpoint function, since others are finite by power counting. It is easy to see that $\sigma^{2}$ and $\Gamma_{\mu \nu} \Gamma_{\mu \nu}$ are the only allowed terms of $\mathcal{O}\left(p^{4}\right)$ consistent with chiral symmetry; since $\Gamma_{\mu}$ transforms as a gauge field, it can only appear as $\Gamma_{\mu \nu}$, where $\Gamma_{\mu v}$ is the field strength (details of transformation properties are discussed in Section IIIc). Thus the local counterterm lagrangian is of the form

$$
A \mathscr{L}_{0}=a \sigma^{2}+b \Gamma_{\mu v} \Gamma_{\mu v}
$$

Since terms like $\sigma^{\prime} \Gamma^{2}$ are not allowed, we expect diagrams like those in Fig. 2 to cancel, which they do; moreover, diagrams in Fig. 3 give

$$
\begin{aligned}
& a=-\frac{1}{2} \frac{1}{16 \pi^{2}(n-4)} \\
& b=-\frac{1}{12} \frac{1}{16 \pi^{2}(n-4)},
\end{aligned}
$$

where we have used dimensional regularization. This is in agreement with earlier results $[1,6,7]$.
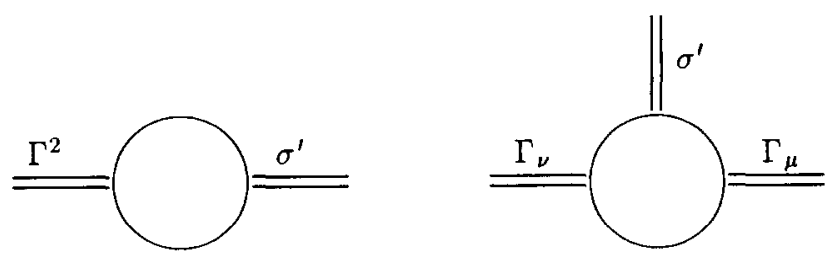

FIG. 2. Vanishing diagrams in the non-anomalous sector by virtue of symmetry. 

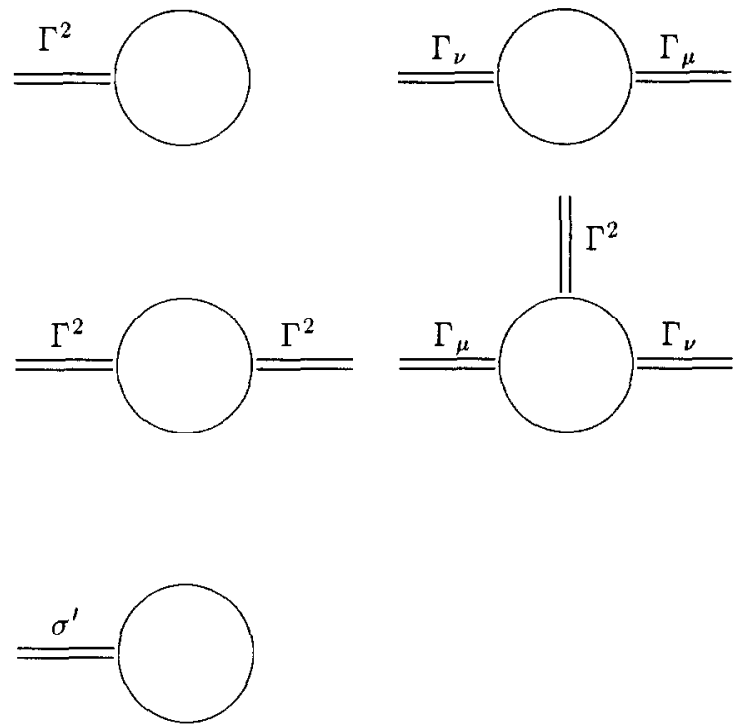

FIG. 3. Diagrams contributing to the one-loop infinities in the non-anomalous sector.

IIIb. Infinities in the Anomalous Sector

In order to check whether the coefficient of the Wess-Zumino term (WZ) is renormalized or not it is necessary to calculate the WZ counter-lagrangian $\Delta \mathscr{L}_{\mathrm{WZ}}$ containing the one-loop divergencies. For simplicity and to illustrate the method, we shall consider first the case without gauge fields. At the end of this section we present the complete result including gauge fields. From Eq. (3.1b) we have, by redefining $\xi \rightarrow f \xi$,

$$
\begin{aligned}
\mathscr{L}_{\mathrm{WZ}}^{(2)}= & \frac{i N_{c}}{12 \pi^{2} f^{2}} \varepsilon^{\mu v \alpha \beta}\left\{\left[\xi^{b} \partial_{\mu} \xi^{a}-\xi^{a} \partial_{\mu} \xi^{b}\right)\right] \frac{1}{2}\left[\left\langle\left(\lambda^{a} \Delta_{v} \lambda^{b}-\lambda^{b} \Delta_{v} \lambda^{a}\right) \Delta_{\alpha} \Delta_{\beta}\right\rangle\right. \\
& \left.\left.-\left\langle\left[\lambda^{a}, \lambda^{b}\right] \Delta_{v} \Delta_{\alpha} \Delta_{\beta}\right\rangle\right]+\xi^{a} \xi^{b}\left\langle\left[\Gamma_{\mu}, \lambda^{a}\right]\left\{\left[\Delta_{v}, \lambda^{b}\right], \Delta_{\alpha} \Delta_{\beta}\right\}\right\rangle\right\}
\end{aligned}
$$

which gives the two vertices in Fig. 4. Here, $\Gamma_{\mu}$ is defined in IIIc and $S_{\mu}^{a b}\left(p_{1}, p_{2}\right)$ is the Fourier transform of

$$
\begin{aligned}
S_{\mu}^{a b}= & \frac{i N_{c}}{12 \pi^{2} f^{2}} \varepsilon^{\mu v \alpha \beta}\left\{\left\langle\left(\lambda^{a} \Delta_{v} \lambda^{b}-\lambda^{b} \Delta_{v} \lambda^{a}\right) \Delta_{\alpha} \Delta_{\beta}\right\rangle\right. \\
& \left.-\left\langle\left[\lambda^{a}, \lambda^{b}\right] \Delta_{v} \Delta_{\alpha} \Delta_{\beta}\right\rangle\right\} .
\end{aligned}
$$

Note that $S_{\mu}$ transforms covariantly under the chiral transformations. 

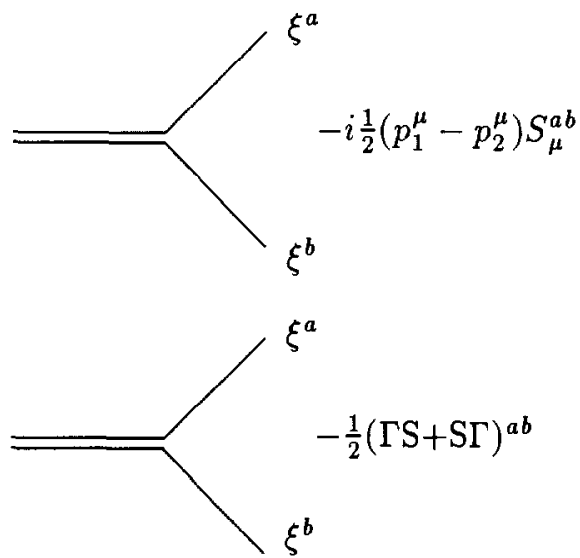

FIG. 4. Feynman rules for the anomalous sector without gauge fields.

To calculate $\Delta \mathscr{L}_{\mathrm{WZ}}$ we have to consider all diagrams with one $\mathrm{WZ}$ vertex and one or more non-anomalous vertices. Moreover, five and higher point functions are finite by power counting and need not be considered. The same power counting argument shows that $\Delta \mathscr{L}_{\mathrm{wz}}$ is $\mathcal{O}\left(p^{6}\right)$. From symmetry considerations, $\Delta \mathscr{L}_{\mathrm{w} 7}$ has to be of the form

$$
\Delta \mathscr{L}_{\mathrm{WZ}}^{\infty}=c\left\langle\Gamma_{\mu \nu}\left(d_{\mu} S_{v}-d_{v} S_{\mu}\right)\right\rangle .
$$

Although $S_{\mu} S_{\mu}$ is $\mathcal{O}\left(p^{6}\right)$ and consistent with symmetries, it is ruled out in (3.7), since $\Delta \mathscr{L}_{\mathrm{wz}}$ has to be proportional to $\varepsilon^{\mu v \alpha \beta}$, Furthermore, terms like $S_{\mu} \Gamma_{\mu} \sigma^{\prime}$ are not allowed because $\Gamma_{\mu}$ has to appear in the form of $\Gamma_{\mu \nu}$ (see arguments in IIIa). Thus, the infinite part of diagrams in Fig. 5 are expected to cancel, which they do.

Using dimensional regularization to calculate the infinite parts of diagrams in Fig. 6, we find

$$
c=-\frac{1}{6} \frac{1}{16 \pi^{2}(n-4)} .
$$

Using the identities

$$
\begin{aligned}
\Gamma_{\mu v} & =-\left[\Delta_{\mu}, \Delta_{v}\right] \quad \text { (no gauge fields) } \\
\sum_{a=1}^{N^{2}-1}\left\langle\lambda^{a} A \lambda^{a} B\right\rangle & =-\frac{2}{N}\langle A B\rangle+2\langle A\rangle\langle B\rangle
\end{aligned}
$$

and

$$
\sum_{a=1}^{N^{2}-1}\left\langle\lambda^{a} A\right\rangle\left\langle\lambda^{a} B\right\rangle=2\langle A B\rangle-\frac{2}{N}\langle A\rangle\langle B\rangle
$$



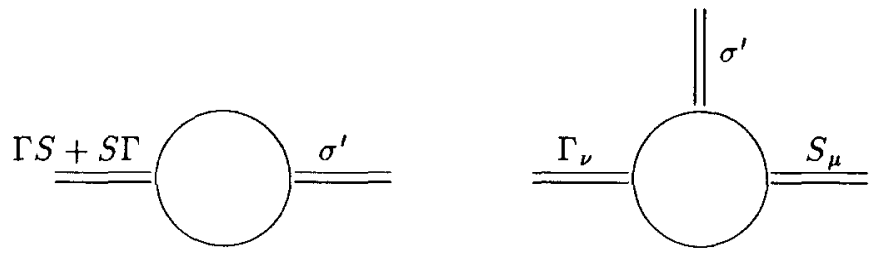

FIG. 5. Vanishing diagrams in the anomalous sector by virtue of symmetry.

which follow from the $S U(N)$ completeness relation

$$
\sum_{a=1}^{N^{2}-1} \lambda_{i j}^{a} \lambda_{k l}^{a}=2\left(\delta_{i}^{l} \delta_{k}^{j}-\frac{1}{N} \delta_{i}^{j} \delta_{k}^{l}\right)
$$

we can write $\Delta \mathscr{L}_{\mathrm{wZ}}^{\infty}$ as

$$
\begin{aligned}
\Delta \mathscr{L}_{\mathrm{wZ}}^{\infty}= & \frac{1}{16 \pi^{2}(n-4)} \frac{-i N_{c}}{96 \pi^{2} f^{2}} \varepsilon^{\mu v \alpha \beta} \\
& \times\left\{-\frac{8 N}{3} 4\left[2\left\langle d_{\lambda} \Delta_{\lambda} \Delta_{\mu} \Delta_{v} \Delta_{\alpha} \Delta_{\beta}\right\rangle+\left\langle d_{\lambda} \Delta_{\mu}\left[\Delta_{v} \Delta_{\alpha} \Delta_{\beta}, \Delta_{\lambda}\right]\right\rangle\right]\right\},
\end{aligned}
$$

where $N_{c}$ and $N$ are respectively the numbers of color and flavors $(N=3$ for chiral $S U(3))$.

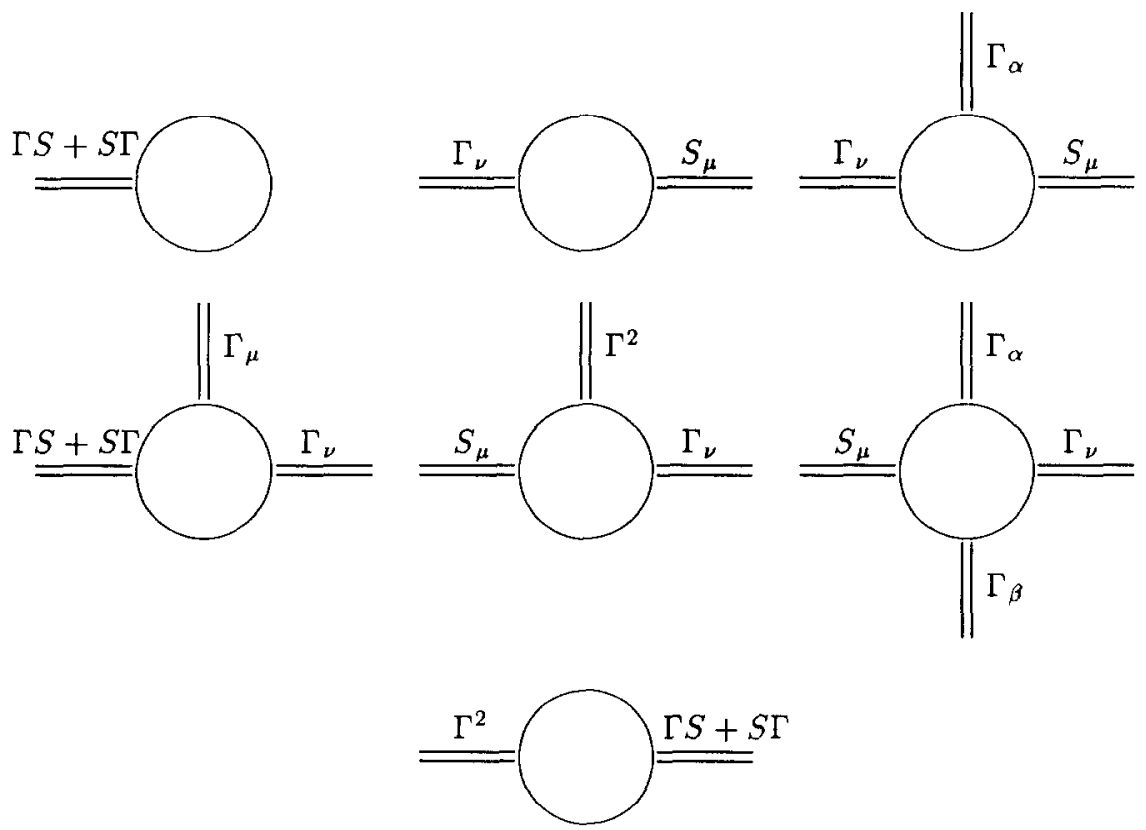

FiG. 6. Diagrams contributing to the one-loop infinities in the anomalous sector without gauge fields. 
The calculation of $\Delta \mathscr{L}_{\mathrm{wZ}}^{\infty}$ including gauge fields proceeds in an analogous manner. In contrast to the case without gauge fields, here there is a term $\sigma_{\mathrm{wz}}$ that is independent of derivatives in the lagrangian for the quadratic fluctuations. The result of a calculation including the gauge fields gives

$$
\Delta \mathscr{L}_{\mathrm{WZ}}^{\infty}=\frac{1}{16 \pi^{2}(n-4)}\left(-\frac{1}{6}\left\langle\Gamma_{\mu v}\left(d_{\mu} S_{v}^{\prime}-d_{v} S_{\mu}^{\prime}\right)-\sigma_{\mathrm{wZ}} \sigma\right\rangle\right)
$$

where

$$
\begin{aligned}
S_{\mu}^{\prime}{ }^{u}= & \frac{i N_{c}}{48 \pi^{2} f^{2}} \varepsilon^{\mu v \alpha \beta}\left\langle\left\{\left[\lambda^{a}, \lambda^{b}\right]\left\{I_{v \alpha}, \Delta_{\beta}\right\}\right.\right. \\
& \left.\left.+\left(\lambda^{a} \Delta_{v} \lambda^{b}-\lambda^{b} \Delta_{v} \lambda^{a}\right)\left(-\Gamma_{\alpha \beta}+2 \Delta_{\alpha} \Delta_{\beta}\right)\right\}\right\rangle
\end{aligned}
$$

and

$$
\begin{aligned}
\sigma_{\mathrm{WZW}}^{a b}= & \frac{i N_{c}}{96 \pi^{2} f^{2}} \varepsilon^{\mu \nu \alpha \beta}\left\langle\left\{\left\{\lambda^{a}, \lambda^{b}\right\}\left(\frac{1}{4}\left[F_{\mu \nu}^{-}, \Gamma_{\alpha \beta}\right]-\frac{1}{2}\left[F_{\mu \nu}^{-}, \Delta_{\alpha} \Delta_{\beta}\right]\right)\right.\right. \\
& \left.\left.-\left(\lambda^{a} \Delta_{\mu} \lambda^{b}+\lambda^{b} \Delta_{\mu} \lambda^{a}\right)\left\{\Delta_{v}, F_{\alpha \beta}^{-}\right\}\right\}\right\rangle .
\end{aligned}
$$

Using identities (3.8b) and (3.8c) and in the case of gauge fields,

$$
\Gamma_{\mu \nu}=-\left[\Delta_{\mu}, \Delta_{\nu}\right]-\frac{1}{2} F_{\mu \nu}^{+},
$$

we can write $(3.10)$ as

$$
\begin{aligned}
\Delta \mathscr{L}_{\mathrm{WZ}}^{\infty}= & \frac{1}{16 \pi^{2}(n-4)} \frac{-i N_{c}}{96 \pi^{2} f^{2}} \varepsilon^{\mu v \alpha \beta} \\
& \times\left\{-\frac{8 N}{3}\left\langle d_{\lambda} \Gamma_{\lambda \mu}\left\{\Gamma_{v \alpha}, \Delta_{\beta}\right\}\right\rangle+\frac{N^{2}-4}{2 N}\left\langle M^{+}\left[\Gamma_{\mu v}, F_{\alpha \beta}^{-}\right]\right\rangle\right. \\
& -N\left\langle M^{+}\left[\Delta_{\mu} \Delta_{v}, F_{\alpha \beta}^{-}\right]\right\rangle-N\left\langle\Delta_{\lambda} \Delta_{\lambda}\left[\Gamma_{\mu \nu}, F_{\alpha \beta}^{-}\right]\right\rangle \\
& +2 N\left\langle\Delta_{\lambda} \Delta_{\lambda}\left[\Delta_{\mu} \Delta_{v}, F_{\alpha \beta}^{-}\right]\right\rangle+4\left\langle M^{+} \Delta_{\mu}\right\rangle\left\langle\Delta_{v} F_{\alpha \beta}^{-}\right\rangle \\
& \left.-8\left\langle\Delta_{\lambda} \Delta_{\lambda} \Delta_{\mu}\right\rangle\left\langle\Delta_{v} F_{\alpha \beta}^{-}\right\rangle+8\left\langle\Delta_{\lambda} \Delta_{\mu}\right\rangle\left\langle\left\{\Delta_{v}, \Delta_{\lambda}\right\} F_{\alpha \beta}^{-}\right\rangle\right\},
\end{aligned}
$$

where,

$$
\begin{aligned}
d_{\alpha} \Gamma_{\mu \nu}= & \partial_{\alpha} \Gamma_{\mu \nu}+\left[\Gamma_{\alpha}, \Gamma_{\mu \nu}\right] \\
= & -\left[d_{\alpha} \Delta_{\mu}, \Delta_{v}\right]-\left[\Delta_{\mu}, d_{\alpha} \Delta_{\nu}\right] \\
& -\frac{1}{2}\left[\Delta_{\alpha}, F_{\mu \nu}^{-}\right]-\frac{1}{2} i\left(u D_{\alpha} F_{\mu \nu}^{L} u^{\dagger}+u^{\dagger} D_{\alpha} F_{\mu \nu}^{R} u\right), \\
D_{\alpha} F_{\mu \nu}^{L, R}= & \partial_{\alpha} F_{\mu \nu}^{L, R}-i\left[F_{\alpha}^{L, R}, F_{\mu \nu}^{L, R}\right] .
\end{aligned}
$$


Here, a few comments are in order: first, all terms of $\Delta \mathscr{L}_{\mathrm{wZ}}^{\infty}$ are seen to be made up of $A_{\mu}, \Gamma_{\mu \nu}, F_{\mu \nu}^{-}, M^{ \pm}$, and $d_{\mu} \Gamma_{\nu \alpha}$. In fact, this is expected since $\mathscr{L}^{(2)}$, (3.1) after integrating out $\xi$ 's is a function of these quantities only; moreover, this is true for all powers of the quantum fluctuation field $\xi$ and not just for the quadratic ones as discussed in general in Section II. Thus, in working out the counterterms to any loop order we can build up the most general lagrangian out of $\Delta_{\mu}, \Gamma_{\mu v}, F_{\mu \nu}^{-}, M^{ \pm}$, and their covariant derivations up to the required order in momenta and masses. Indeed, in Section 3.3 using this procedure, we shall give the complete $\mathcal{O}\left(p^{6}\right)$ counter-lagrangian in the anomalous sector. Second, none of the terms in (3.11) is identical to the tree level WZ effective lagrangian, which explicitly shows the non-renormalizability of $\mathrm{WZ}$ term at the one-loop level. The infinities of the $\mathcal{O}\left(p^{6}\right)$ lagrangian have also been discussed in $[6,7]$ in a different basis.

\section{IIIc. The Complete Anomalous Counter Lagrangian to $\mathcal{O}\left(p^{6}\right)$}

In the previous subsection we saw that $\Delta_{\mu}, \Gamma_{\mu \nu}, F_{\mu \nu}^{-}, M^{+}, M^{-}$, and their corresponding covariant derivatives can be considered as building blocks for constructing the WZ counter-lagrangian to any order in a consistent momentum expansion. The complete $\mathrm{WZ}$ counter-lagrangian which is $\mathcal{O}\left(p^{6}\right)$ is presented in this section; details of its construction can be found in Appendix I. We first need to list the transformation properties of the above objects under $S U(3)_{L} \times S U(3)_{R}$ and its diagonal subgroup $S U(3)_{V}$. Under a chiral transformation,

$$
\begin{aligned}
U & \rightarrow V_{R} U V_{L}^{\dagger} \\
u & \rightarrow V_{R} u h^{\dagger}=h u V_{L}^{\dagger} \\
u^{\dagger} & \rightarrow h u^{\dagger} V_{R}^{\dagger}=V_{L} u^{\dagger} h^{\dagger},
\end{aligned}
$$

where $h \in S U_{V}(3)$. Under the vectorial subgroup $S U_{V}(3)$,

$$
\begin{aligned}
\Delta_{\mu} & \rightarrow h \Delta_{\mu} h^{\dagger} \\
F_{\mu \nu}^{-} & \rightarrow h F_{\mu \nu}^{-} h^{\dagger} \\
\Gamma_{\mu} & \rightarrow h \Gamma_{\mu} h^{\dagger}+h \partial_{\mu} h^{\dagger} ;
\end{aligned}
$$

thus $\Gamma_{\mu}$ transforms as a connection. The corresponding field strength tensor $\Gamma_{\mu \nu}=\partial_{\mu} \Gamma_{\nu}-\partial_{\mu} \Gamma_{\mu}+\left[\Gamma_{\mu}, \Gamma_{v}\right]$ transforms as

$$
\Gamma_{\mu v} \rightarrow h \Gamma_{\mu \nu} h^{\dagger}
$$

Since $M^{+}$breaks explicitly the chiral symmetry, it is considered as a spurion and it transforms, under $h \in S U_{V}(3)$, as

$$
M^{ \pm} \rightarrow h M^{ \pm} h^{\dagger} .
$$


Next, we consider the parity and change conjugation transformation,

$$
\begin{gathered}
u(\mathbf{x}, t) \stackrel{P}{\longrightarrow} u^{\dagger}(-\mathbf{x}, t) \\
F_{\mu}^{L}(\mathbf{x}, t) \stackrel{P}{\longrightarrow}-F_{\mu}^{R}(-\mathbf{x}, t) \delta(\mu),
\end{gathered}
$$

where

$$
\begin{aligned}
& \delta(\mu)=\left\{\begin{aligned}
-1 & \text { for } \quad \mu=0 \\
1 & \text { for } \quad \mu=1,2,3
\end{aligned}\right. \\
& u \stackrel{C}{\longrightarrow} u^{t} \\
& F_{\mu}^{R} \stackrel{C}{\longrightarrow}-F_{\mu}^{R t} \quad(t \text { denotes transpose }) .
\end{aligned}
$$

The transformation properties of $\Delta_{\mu}, d_{\mu} \Delta_{v}, \Gamma_{\mu \nu}, d_{\mu} \Gamma_{v \alpha}, F_{\mu \nu}^{-}, d_{\mu} F_{v \alpha}^{-}$, and $M^{ \pm}$can now be easily deduced and are tabulated below:

\begin{tabular}{ccc} 
Quantity & $P$ & $\mathrm{C}$ \\
\hline$\Delta_{\mu}$ & $\Delta_{\mu} \delta(\mu)$ & $\Delta_{\mu}^{t}$ \\
$d_{\mu} \Delta_{v}$ & $-d_{\mu} \Delta_{v} \delta(\mu) \delta(v)$ & $d_{\mu} \Delta_{v}^{t}$ \\
$\Gamma_{\mu v}$ & $\Gamma_{\mu \nu} \delta(\mu) \delta(v)$ & $-\Gamma_{\mu v}^{t}$ \\
$d_{\lambda} \Gamma_{\mu v}$ & $-d_{\lambda} \Gamma_{\mu \nu} \delta(\lambda) \delta(\mu) \delta(v)$ & $-d_{\lambda} \Gamma_{\mu v}^{t}$ \\
$F_{\mu v}^{-}$ & $-F_{\mu \nu}^{-} \delta(\mu) \delta(v)$ & $F_{\mu v}^{-t}$ \\
$d_{\lambda} F_{\mu v}^{-}$ & $d_{\lambda} F_{\mu v}^{-} \delta(\lambda) \delta(\mu) \delta(v)$ & $d_{\lambda} F_{\mu v}^{\mu t}$ \\
$M^{ \pm}$ & $\pm M^{ \pm}$ & $M^{ \pm}$ \\
\hline
\end{tabular}

Notice that $\Delta_{\mu}, d_{\mu} \Delta_{v}, \Gamma_{\mu \nu}, d_{\lambda} \Gamma_{\mu v}, F_{\mu \nu}^{-}, d_{\lambda} F_{\mu v}^{-}, M^{-}$are anti-hermitian, whereas $M^{+}$is hermitian. Furthermore, we have included only the first covariant derivative, since as we shall see in Appendix I, second and higher order derivatives are not needed to the order that we are considering. The most general lagrangian consistent with chiral symmetry, $P$, and $C$ containing, the totally anti-symmetric $\varepsilon$-tensor and which is $\mathcal{O}\left(p^{6}\right)$ is

$$
\begin{aligned}
\mathscr{L}_{(6)}= & i \varepsilon^{\mu \nu \alpha \beta}\left\{w_{1}\left\langle F_{\beta \lambda}^{-}\left\{\Gamma_{\mu \nu}, \Gamma_{\alpha \lambda}\right\}\right\rangle\right. \\
& +w_{2}\left\langle d_{\lambda} F_{\lambda \mu}^{-}\left\{F_{\alpha \beta}^{-}, \Delta_{v}\right\}\right\rangle \\
& +w_{3}\left\langle d_{\lambda} F_{\mu \nu}^{-}\left\{F_{\lambda \beta}^{-}, \Delta_{\alpha}\right\}\right\rangle \\
& +w_{4}\left\langle d_{\lambda} \Gamma_{\lambda \mu}\left\{\Gamma_{\alpha \beta}, \Delta_{\nu}\right\}\right\rangle \\
& +w_{5}\left\langle d_{\lambda} \Gamma_{\mu \nu}\left\{\Gamma_{\lambda \beta}, \Delta_{\alpha}\right\}\right\rangle \\
& +w_{6}\left\langle d_{\lambda} F_{\alpha \beta}^{-}\left\{d_{\lambda} \Delta_{\mu}, \Delta_{v}\right\}\right\rangle
\end{aligned}
$$




$$
\begin{aligned}
& +w_{7}\left\langle\Delta_{\lambda} \Delta_{\lambda}\left[\Gamma_{\mu v}, F_{\alpha \beta}^{-}\right]\right\rangle \\
& +w_{8}\left\langle\Delta_{\lambda} \Delta_{\mu} \Gamma_{v \alpha} F_{\overline{\beta \lambda}}-\Delta_{\mu} \Delta_{\lambda} F_{\overline{\beta \lambda}} \Gamma_{v \alpha}\right\rangle \\
& +w_{9}\left\langle\Delta_{\mu} \Delta_{\lambda} \Gamma_{v \alpha} F_{\bar{\beta}}^{-}-\Delta_{\hat{\lambda}} \Delta_{\mu} F_{\beta \bar{\lambda}}^{-} \Gamma_{v \alpha}\right\rangle \\
& +w_{10}\left\langle\Delta_{\mu} \Delta_{\nu}\left\{\Gamma_{\alpha \lambda}, F_{\bar{\beta} i}\right\}\right\rangle \\
& +w_{11}\left\langle\Delta_{\lambda} \Gamma_{\lambda v} \Delta_{\mu} F_{\alpha \beta}^{-}-\Delta_{\mu} \Gamma_{v \lambda} \Delta_{\lambda} F_{\alpha \beta}^{-}\right\rangle \\
& +w_{12}\left\langle\Delta_{\lambda} \Gamma_{v \alpha} \Delta_{\mu} F_{\beta \lambda}^{-}-\Delta_{\mu} \Gamma_{v \alpha} \Delta_{\lambda} F_{\beta \lambda}^{-}\right\rangle \\
& +w_{13}\left\langle\Delta_{\mu} \Gamma_{\alpha \lambda} \Delta_{v} F_{\beta \lambda}^{-}\right\rangle \\
& +w_{14}\left\langle\Delta_{\mu} \Delta_{v}\left\{\Gamma_{\alpha \beta}, d_{\lambda} \Delta_{i}\right\}\right\rangle \\
& +w_{1 S}\left\langle\Delta_{v} \Delta_{\lambda} \Gamma_{\alpha \beta} d_{i} \Delta_{\mu}-\Delta_{\lambda} \Delta_{v} d_{\lambda} \Delta_{\mu} \Gamma_{\alpha \beta}\right\rangle \\
& +w_{16}\left\langle\Delta_{v} \Delta_{\alpha}\left\{\Gamma_{\beta \lambda}, d_{\lambda} \Delta_{\mu}\right\}\right\rangle \\
& +w_{17}\left\langle\Delta_{\mu} d_{\lambda} \Delta_{\lambda} \Delta_{v} \Gamma_{\alpha \beta}\right\rangle \\
& +w_{18}\left\langle\Delta_{v} d_{\lambda} \Delta_{\mu} \Delta_{\alpha} \Gamma_{\beta \lambda}\right\rangle \\
& +w_{19}\left\langle F_{\mu \nu}^{-}\left(\Delta_{\alpha} \Delta_{\beta} \Delta_{\lambda} \Delta_{\lambda}+\Delta_{\lambda} \Delta_{\lambda} \Delta_{\beta} \Delta_{\alpha}\right)\right\rangle \\
& +w_{20}\left\langle F_{\mu \lambda}\left(\Delta_{v} \Delta_{\alpha} \Delta_{\beta} \Delta_{\lambda}+\Delta_{\lambda} \Delta_{\beta} \Delta_{\alpha} \Delta_{v}\right)\right\rangle \\
& +w_{21}\left\langle F_{\mu v}^{-} \Delta_{\alpha}\right\rangle\left\langle\Delta_{\beta} \Delta_{\lambda} \Delta_{\lambda}\right\rangle \\
& +w_{22}\left\langle F_{\mu \nu}^{-}\left\{\Delta_{\alpha}, \Delta_{\lambda}\right\}\right\rangle\left\langle\Delta_{\lambda} \Delta_{\beta}\right\rangle \\
& +w_{23}\left\langle d_{\lambda} \Delta_{\lambda} \Delta_{\mu} \Delta_{v} \Delta_{\alpha} \Delta_{\beta}\right\rangle \\
& +w_{24}\left\langle d_{\lambda} \Delta_{\mu}\left[\Delta_{v} \Delta_{\alpha} \Delta_{\beta}, \Delta_{\lambda}\right]\right\rangle \\
& +w_{25}\left\langle M^{+}\left[\Gamma_{j w}, F_{\alpha \beta}^{-}\right]\right\rangle \\
& +w_{26}\left\langle M^{+}\left[F_{\alpha \beta}^{-}, \Delta_{\mu} \Delta_{v}\right]\right. \\
& +w_{27}\left\langle M^{+} \Delta_{\mu}\right\rangle\left\langle\Delta_{v} F_{x \beta}^{-}\right\rangle \\
& +w_{28}\left\langle M^{-}\right\rangle\left\langle F_{\mu \nu}^{-} F_{\alpha \beta}^{-}\right\rangle \\
& +w_{29}\langle M\rangle\left\langle\Gamma_{\mu \nu} \Gamma_{\alpha \beta}\right\rangle \\
& \left.+w_{30}\left\langle M^{-}\right\rangle\left\langle\Delta_{\mu} \Delta_{v} \Gamma_{x \beta}\right\rangle\right\} \text {. }
\end{aligned}
$$

In the above, we assume that the background fields satisfy the equation of motion, (2.18) (relevant to this order).

There are thus 30 independent real constants at this level $\left(w_{1}, \ldots, w_{30}\right)$ in contrast to the 10 constants at the $\mathcal{O}\left(p^{4}\right)$ level found by Gasser and Leutwyler [1]. The details of the construction are given in Appendix I, and the various ideas used are invariance arguments, integration by parts, the Shouten identity, etc. Comparing with the results in (3.11), we see that the coefficients $w_{4}, w_{7}, w_{19}, w_{21}, w_{22}, w_{25}$, 
$w_{26}$, and $w_{27}$ are infinite, whereas the rest are finite in the basis that we have chosen. As an interesting special case, consider the pure hadronic anomaly by setting all gauge fields to zero. $F_{\mu \nu}^{-}$vanishes; furthermore $\Gamma_{\mu v}$ reduces to $\Gamma_{\mu \nu}=-\left[\Delta_{\mu}, \Delta_{v}\right]$; hence the only terms needed in constructing the counter-lagrangian are $\Delta_{\mu}$ and $d_{\mu} \Delta_{v}$. Thus the only independent terms would be $w_{23}$ and $w_{24}$ and both of them, in this case, are infinite. Finally, we note that if the equation of motion (2.18) is not used there are five additional terms in Eq. (3.12):

$$
\begin{aligned}
i \varepsilon^{\mu \nu \alpha \beta} & \left\{w_{31}\left\langle M^{-} F_{\mu \nu}^{-} F_{\alpha \beta}^{-}\right\rangle\right. \\
& +w_{32}\left\langle M^{-} \Gamma_{\mu \nu} \Gamma_{\alpha \beta}\right\rangle \\
& +w_{33}\left\langle\Delta_{\mu} \Delta_{\nu}\left\{\Gamma_{\alpha \beta}, M^{-}\right\}\right\rangle \\
& +w_{34}\left\langle M^{-} \Delta_{\mu} \Delta_{\nu} \Delta_{\alpha} \Delta_{\beta}\right\rangle \\
& \left.+w_{35}\left\langle M^{-} \Delta_{\mu} \Gamma_{\nu \alpha} \Delta_{\beta}\right\rangle\right\} .
\end{aligned}
$$

\section{Higher Order Terms in Chiral Perturbation Theory}

We begin this section with a discussion of the two-dimensional Wess-Zumino model to all orders in the background field expansion. We will consider only the conformally invariant case $\kappa^{2}=1$, because as we shall see an analytic proof for the non-renormalization of the Wess-Zumino term can be given for this case. The action incorporating the fluctuations to all orders was obtained in Section IIb, i.e., (2.27). We would first like to express this action in a more symmetric manner, which is possible in two dimensions with $\kappa^{2}=1$. In order to do this, the following identities relevant for this case are important: For a vector, $X^{\mu}$,

$$
\begin{aligned}
& \left(g^{\mu v} \pm \varepsilon^{\mu v}\right)\left(g_{\mu x} \pm \varepsilon_{\mu x}\right) X^{\alpha}=0 \\
& \left(g^{\mu v} \pm \varepsilon^{\mu v}\right)\left(g_{v \beta} \pm \varepsilon_{v \beta}\right) X^{\beta}=2\left(g^{\mu v} \pm \varepsilon^{\mu v}\right) X_{v} .
\end{aligned}
$$

Using $(4.1)$, we may rewrite $(2.27 \mathrm{~b})$ as

$$
\begin{aligned}
S_{\mathrm{WZ}}(1)-\left.S_{\mathrm{WZ}}(0)\right|_{2 D}= & \frac{f^{2}}{2}\left(g^{\mu v} \pm \varepsilon^{\mu v}\right) \int_{0}^{1} d t \int_{0} d s \int d^{2} x \\
& \times\left\{\left\langlee^{-i \xi s}\left(\partial_{\mu} \xi+\left(g_{\mu \alpha} \pm \varepsilon_{\mu \alpha}\right)\left[u^{-1} \Delta^{\alpha} u, \xi\right]\right) e^{i \xi_{s}}\right.\right. \\
& \left.\left.\times\left(\partial_{v} \tilde{\xi}+\left(g_{v \beta} \pm \varepsilon_{v \beta}\right)\left[u^{-1} \Delta^{\beta} u, \xi\right]\right)\right\rangle\right\} .
\end{aligned}
$$

We may now notice that $\left(g_{\mu \alpha} \pm \varepsilon_{\mu \alpha}\right) u^{-1} \Delta^{\alpha} u$ is a pure gauge, i.e., we may write

$$
\left(g_{\mu \alpha} \pm \varepsilon_{\mu \alpha}\right) u^{-1} \Delta^{\alpha} u=k_{\mu} .
$$

Then, $\varepsilon^{\mu v}\left(\partial_{\mu} k_{v}+k_{\mu} k_{v}\right)=0\left(d k=-k^{2}\right)$ and $k_{\mu}=V^{-1} \partial_{\mu} V$. 
In view of this, we may write (4.2) as

$$
S_{\mathrm{WZ}}(1)-\left.S_{\mathrm{WZ}}(0)\right|_{2 D}=\frac{f^{2}}{2}\left(g^{\mu \nu} \pm \varepsilon^{\mu v}\right) \int_{0}^{1} d t \int_{0}^{t} d s \int d^{2} x\left\{\left\langle e^{-i \tilde{\xi}_{s}} \partial_{\mu} \tilde{\xi}^{i \tilde{\xi}_{s}} \partial_{v} \tilde{\xi}\right\rangle\right\}
$$

with $\tilde{\xi}=V \tilde{\xi} V^{-1}=V u^{-1} \xi u V^{-1}$.

We now make a change of variables $\xi \rightarrow \widetilde{\xi}$ in (2.36) for which the measure is invariant and in this way all reference to the background fields may be eliminated from Eq. (4.4). Clearly then, the Wess-Zumino term is unrenormalized to all orders. For other discussions of the two-dimensional Wess-Zumino model see [8-10].

We next proceed to a discussion of the four-dimensional case using the background field method discussed in Section II. The first remark we would like to emphasize is that our background field expansion allows us to express the action incorporating the fluctuations, i.e., the term $[S(U(t=1), \ldots-S(\bar{U}, \ldots)]$ in $(2.36)$ purely as a compact form in four dimensional in terms of the fundamental quantities $\Gamma_{\mu}, \Delta_{\mu}, \Gamma_{\mu \nu}, F_{\mu \nu}^{-}$. The procedure outlined at the end of Section II for calculating higher loop diagrams is the standard Dyson expansion and therefore all the local counterterms introduced in higher orders will be expressible in a compact form in four dimensions in terms of chiral invariant structures constructed out of the fundamental quantities. Thus, no local counterterms which are expressible in a compact form (in terms of the $U$ field) only in five dimensions will be introduced. In this connection, it is worth recalling that the purely hadronic Wess-Zumino term (i.e., the one which survives when all external gauge fields are set to zero) has the property that it can only be expressed in compact form in terms of the chiral field $U$ in five dimensions. From this it is reasonable to conclude that in our framework, the coefficient of the Wess-Zumino term will not be renormalized. However, to make this more precise we will present an argument, not in terms of the chiral fields $U$, but rather in terms of the pseudoscalar meson fields $\phi(x)$.

We first consider the situation in the chiral limit, without external gauge fields. The fundamental fact which is responsible for the nonrenormalization of the Wess-Zumino term is that our background field expansion respects parity. In particular, it is easy to check from (2.15a) and (2.25a) that in the non-anomalous sector, in the background field expansion of the action, even powers of $\xi$ come with even powers of $\Delta_{\mu}$ and odd with odd. In the anomalous sector, the situation is reversed; odd powers of $\xi$ come with even powers of $A_{\mu}$ and vice versa. This is a consequence of parity invariance with $\xi$ transforming appropriately. In fact, this is true not just for the lowest order lagrangians in the anomalous and nonanomalous sectors but for all anomalous and nonanomalous interactions. An immediate consequence of this is that terms like $(\partial \phi \partial \phi)^{n}$ and $(d \xi d \xi)^{n}$ appear only in the even order and mixed terms like $(\partial \phi)^{n}(d \xi)^{m}$ appear only in the odd order in the expansions of the nonanomalous interactions. In the expansions in the anomalous sector one has the reverse situation: mixed terms like $(\partial \phi)^{n}(d \xi)^{m}$ appear in the even order and $(\partial \phi \partial \phi)^{n}$ and $(d \xi d \xi)^{n}$ appear in the odd order. It is this, together with the fact that $d_{[\mu} d_{v]}=0$, that is responsible for the non-renormalization theorem. 
In order to check the non-renormalization theorem one has to determine if a counterterm of the form $A_{a b c d}(\phi) \varepsilon^{\mu \nu \alpha \beta} \partial_{\mu} \phi^{a} \partial_{\nu} \phi^{b} \partial_{\alpha} \phi^{c} \partial_{\beta} \phi^{d}$, where $A_{a b c d}(\phi)$ is antisymmetric in its indices, is even induced. If this were the case then the Wess-Zumino term would be renormalized. Consider first for simplicity the situation at the one-loop level. Infinite counterterms of the type mentioned above can come from Feynman diagrams which have a following combination of vertices:

(a) a vertex with $\varepsilon^{\mu v \alpha \beta} \partial_{\mu} \phi^{a} \partial_{v} \phi^{b} \partial_{\rho} \phi^{c} d_{\sigma} \xi^{d}$, together with a vertex with $d_{\mu} \xi^{a} \partial^{\mu} \phi^{b}$ appearing in it.

(b) a vertex with $\varepsilon^{\mu v \alpha \beta} \partial_{\mu} \phi^{a} \partial_{\nu} \phi^{b} \partial_{\rho} \phi^{c} \partial_{\sigma} \phi^{d}$ together with a vertex with $d_{\mu} \xi^{a} d^{\mu} \xi^{b}$

In (a) a vertex like $d_{\mu} \xi^{a} d_{\mu} \phi^{b}$ can come from the non-anomalous sector only if there are terms of the type $d_{\mu} \xi\left[\Delta^{\mu}, \xi\right]$ at the one loop level, i.e., odd powers of $\Delta$ with even powers of $\xi$. Such terms are forbidden in our expansion, so (a) is not possible. In (b) a vertex like $\varepsilon^{\mu \nu \alpha \beta} \partial_{\mu} \phi^{a} \ldots \partial_{\mu} \phi^{d}$ can come only from a term in the anomalous sector of the form $\Delta^{4} \xi^{2}$, i.e., even in $\Delta$ and $\xi$, which is forbidden. Thus, we see that there can be no renormalization of the Wess-Zumino term at the oneloop level as we have explicitly verified. Inclusion of mass terms does not alter the argument since even powers of $\xi$ come with $\mathrm{M}^{+}$and odd powers of $\xi$ come with $M^{-}$. The only way consistent with parity in order to induce a counterterm of the type mentioned earlier is if the background field action in the anomalous sector had a term of the form $\Delta^{4} \xi^{2}$, which it cannot.

The situation in higher orders is not too different; the same general arguments go through. In addition to (a) and (b), we can also induce Wess-Zumino terms through the following:

(c) a vertex with $\varepsilon^{\mu \nu \alpha \beta} \partial_{\mu} \phi^{a} \partial_{\nu} \phi^{b} d_{\rho} \xi^{c} d_{\sigma} \xi^{d}$, together with a vertex with a term like $\partial_{\mu} \phi^{a} \partial^{\mu} \phi^{b} d^{v} \xi^{c} d_{v} \xi^{d}$

(d) a vertex with a tmer $\varepsilon^{\mu \nu \alpha \beta} \partial_{\mu} \phi^{a} d_{v} \xi^{b} d_{\rho} \xi^{c} d_{\sigma} \xi^{d}$, together with a term like $\partial_{\mu} \phi^{a} d^{\mu} \xi^{b} d^{\mu} \xi^{c} d_{\mu} \xi^{d}$.

Notice that if we restrict ourselves to the background field expansions of the two-derivative lagrangian and the $\mathcal{O}\left(p^{4}\right)$ Wess-Zumino lagrangian, terms from the non-anomalous sector needed in (c) and (d) can only come from multiple insertions, if at all. In of the graphs of the type (a) to (d) we can, of course, have multiple insertions of the type $d^{\mu} \xi^{a} d_{\mu} \xi^{b}$ and an even number of insertions of terms with the $\varepsilon$-tensor. Upon inspection, all of the graphs listed above have an odd number of the fluctuating field $\xi$. Since the $\xi$ come only in loops, we must have an even number of these; hence they are excluded. Similar arguments also exclude the mass insertions. The arguments presented are similar to those discussed for the two-dimensional case in the second of Ref. [9].

Gauge fields may also be included. For the vector case, i.e., $F_{\mu}^{L}=F_{\mu}^{R}$, all the arguments discussed aboe go through unchanged. If, however, $F_{\mu \nu}^{-}$is not zero, then in the background field expansion of the Wess-Zumino action, even powers of $\xi$ 
can come with even powers of $\Delta$, provided they are also accompanied by $F_{\mu \nu}^{-}$. Also in this case $d_{[\mu} \Delta_{v\rfloor}=\frac{1}{2} F_{\mu \nu}^{-}$. The arguments presented above have therefore to be modified, but we shall not discuss it further here.

\section{APPENDIX I}

In this appendix we discuss the principles that go into the construction of the counterterms of $\mathcal{O}\left(p^{6}\right)$ given in Eq. ((3.12) which are allowed by parity $P$ and charge conjugation $C$, chiral symmetry, as well as Lorentz invariance. We begin by writing down all possible terms of $\mathscr{O}\left(p^{6}\right)$ that are consistent with chiral symmetry. $P$ and $C$ invariance are then used to eliminate some of these. For example, terms like

$$
\varepsilon^{\mu v \times \beta}\left\langle d_{\mu} \Delta_{\lambda} d_{v} \Delta_{\lambda} \Gamma_{\alpha \beta}\right\rangle
$$

and

$$
\varepsilon^{\mu \nu \alpha \beta}\left\langle\Delta_{\mu} A_{\lambda}\right\rangle\left\langle\Delta_{\nu} \Delta_{\alpha} d_{\lambda} \Delta_{\beta}\right\rangle
$$

were excluded because they are odd under parity (first term) and under charge conjugation (second term). Hermiticity can also be used as a constraint. For each term conserving $P$ and $C$, say $\left\langle d \Delta F^{-} F^{-}\right\rangle$, we had to consider all possible ways of contracting the Lorentz indices. The number of such possibilities was limited not only by the symmetries of some basic building blocks such as $\Gamma_{\mu \nu}$ and $F_{\mu \nu}^{-}$(being anti-symmetric in $\mu$ and $v$ ), but also due to some identities such as

$$
d_{\mu} \Delta_{v}-d_{v} \Delta_{\mu}=\frac{1}{2} F_{\mu v}^{-} .
$$

Hence, when considering all possible ways of contracting the indices in $\left\langle d \Delta F^{-} F^{-}\right\rangle$, for example, only

$$
\begin{aligned}
& \varepsilon^{\mu \nu \alpha \beta}\left\langle d_{\lambda} \Delta_{\mu} F_{v \alpha}^{-} F_{\lambda \beta}^{-}\right\rangle \\
& \varepsilon^{\mu v \alpha \beta}\left\langle d_{\lambda} \Delta_{\mu} F_{v \lambda} F_{\alpha \beta}^{-}\right\rangle \\
& \varepsilon^{\mu \nu \alpha \beta}\left\langle d_{\lambda} \Delta_{\lambda} F_{\mu \nu}^{-}-\overline{\alpha \beta}\right\rangle
\end{aligned}
$$

need to be considered, since terms having $d_{\mu} A_{\lambda}$ and $d_{\mu} A_{v}$ are not independent by virtue of (A.1) and could be written in terms of (A.2), in addition to terms like $\left\langle F_{\mu \nu}^{-} F_{\lambda \alpha}^{-} F_{\lambda \beta}^{-}\right\rangle$which will be considered separately.

Furthermore, some building blocks are not independent or they even vanish when the indices are contracted in some specific ways. As an example,

$$
\begin{aligned}
\varepsilon^{\mu v \alpha \beta} d_{\mu v \alpha} & =-2 \varepsilon^{\mu v \alpha \beta}\left[A_{\mu}, \Gamma_{v \alpha}\right] \\
\varepsilon^{\mu v \alpha \beta} d_{\mu} F_{v \alpha} & =0,
\end{aligned}
$$


hence, terms like that need not be considered. Further constraints arise from using the Shouten identity. Due to the fact that in four dimensions any tensor $T^{\mu v \alpha \cdots \beta}$ which is totally anti-symmetric in five indices is identically zero, we arrive at the following relation:

$$
\delta^{\mu v} \varepsilon^{\alpha \beta \delta \lambda}-\delta^{\mu \alpha} \varepsilon^{\nu \beta \delta \lambda}-\delta^{\mu \beta} \varepsilon^{\alpha v \delta \lambda}-\delta^{\mu \delta} \varepsilon^{\alpha \beta v \lambda}-\delta^{\mu \lambda} \varepsilon^{\alpha \beta \delta v}=0 .
$$

Because of this relation, many terms which otherwise look independent, in fact, are not. As an example, consider the following terms:

$$
\begin{aligned}
\varepsilon^{\mu \nu \alpha \beta} & \left\langle d_{\mu} \Delta_{\lambda}\left\{d_{\lambda} F_{v \alpha}^{-}, \Delta_{\beta}\right\}\right\rangle \\
& +\varepsilon^{\mu \nu \alpha \beta}\left\langle d_{\mu} \Delta_{\lambda}\left\{d_{v} F_{\lambda \alpha}^{-}, \Delta_{\beta}\right\}\right\rangle \\
& +\varepsilon^{\mu \nu \alpha \beta}\left\langle d_{\mu} \Delta_{\lambda}\left\{d_{v} F_{\alpha \beta}^{-}, \Delta_{\lambda}\right\}\right\rangle \\
& +\varepsilon^{\mu v \alpha \beta}\left\langle d_{\lambda} \Delta_{\lambda}\left\{d_{\mu} F_{v \alpha}^{-}, \Delta_{\beta}\right\}\right\rangle .
\end{aligned}
$$

These may be written as

$$
\left\langle d_{\mu} \Delta_{v}\left\{d_{\alpha} F_{\beta \delta}, \Delta_{\lambda}\right\}\right\rangle\left\{\delta_{v \alpha} \varepsilon^{\mu \beta \delta \lambda}+\delta_{v \beta} \varepsilon^{\mu \alpha \delta \hat{\lambda}}+\delta_{v \lambda} \varepsilon^{\mu \beta \delta \alpha}+\delta_{v \mu} \varepsilon^{\alpha \beta \delta \hat{\lambda}}\right\} .
$$

Now, using (A.3) we can show that

$$
\begin{aligned}
\varepsilon^{\mu \nu \alpha \beta}\left\langle d_{\mu} \Delta_{\lambda}\left\{d_{\lambda} F_{v \alpha}^{-}, \Delta_{\beta}\right\}\right\rangle= & \varepsilon^{\mu v \alpha \beta}\left\{\left\langle d_{\lambda} \Delta_{\lambda}\left\{d_{\mu} F_{v \alpha}^{-}, \Delta_{\beta}\right\}\right\rangle\right. \\
& +2\left\langle d_{\mu} \Delta_{\lambda}\left\{d_{v} F_{\lambda \alpha}^{-}, \Delta_{\beta}\right\}\right\rangle \\
& \left.+\left\langle d_{\mu} \Delta_{\lambda}\left\{d_{v} F_{\alpha \beta}^{-}, \Delta_{\lambda}\right\}\right\rangle\right\} .
\end{aligned}
$$

Finally, terms having higher order derivatives like $d d \Delta, d d d \Delta, \ldots$ are not needed to this order $\left(\mathcal{O}\left(p^{6}\right)\right)$ because they could be reduced to terms with one derivative either by partial integration or by exploiting the relation

$$
\left[d_{\mu}, d_{v}\right] \varnothing=\left[\Gamma_{\mu \nu}, \varnothing\right] \text {. }
$$

Using the ideas discussed above, repeatedly, we arrive at the set of independent operators given in Eq. (3.12).

\section{ACKNOWLEDGMENT}

This work was supported in part by DoE.

Note added in proof. Upon completion of this work, we received a preprint entitled "Nonrenormalization of the Chiral Anomaly in Chiral Perturbation Theory" by D. Issler [SLAC-PUB-4943 Rev.], where some similar issues are discussed. The $\mathcal{O}\left(p^{6}\right)$ chiral lagrangian obtained in Issler's paper is different than the one given in Section IIIc. 


\section{REFERENCES}

1. J. Gasser and H. Leutwyler, Ann. Phys. (N.Y.) 158 (1984), 142; Nucl. Phys. B 250 (1985), 4654.

2. J. Wess And B. Zumino, Phys. Lett. B 37 (1971), 95.

3. E. Witten, Nucl. Phys. B 223 (1983), 422.

4. L. Alvarez-Guamé and P. Ginsparg, Ann. Phys. 161 (1985), 423.

5. C. K. Chau, G. H. Ying, and W. Ke, Phys. Lelt. B 134 (1984), 67; C. Callan and E. Witten, Nucl. Phys. B 239 (1984); H. KaWAl and H. Tye, Phys. Lett. B 140 (1984), 403; J. L. Mañes, Nucl. Phys. B 250 (1985), 369.

6. J. Donoghue and D. Wyler, Nucl. Phys. B 316 (1989), 289.

7. J. Bunens, A. Bramon, and F. Cornet, Z. Phys. C 46 (1990), 599.

8. J. Honerkamp, Nucl, Phys. B (1984), 130; L. Alvarez-Gaumé, D. Freedman, S. Mukhi, Ann. Phys. (N.Y.) 134 (1981), 85; D. Boulware And L. S. Brown, Ann. Phys. (N.Y.) 138 (1982), 392.

9. E. Braaten, T. Curtright, and C. Zachos, Nucl. Phys. B 260 (1985), 630; S. Mukhi, ibid. 264 (1986), 640; B. Fridling and A. VAN de Ven, ibid. 268 (1986), 719.

10. E. Witten, Commun. Math. Phys. 92 (1984), 455; A. Polyakov and P. Weigman, Phys. Lett. B 131 (1983), 1212; 141 (1983), 223; P. Di Vecchia and P. Rossi, Phys. Lett. B 140 (1984), 344.

11. B. S. DEWITT, Phys. Rev. 162 (1967), 1195, 1239.

12. B. S. DeWitt and M. T. Grisaru, Phys. Rev. D 20 (1979), 2082. 